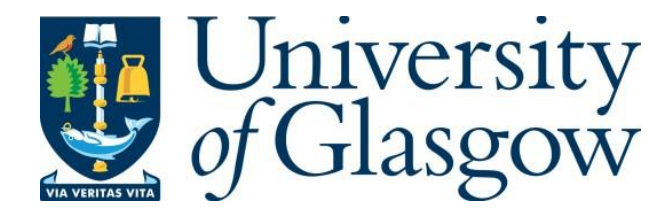

Todorova, B.N. and Steijl, R. (2019) Comparison of discrete velocity method and gas-kinetic method for binary gas mixtures. Journal of Thermophysics and Heat Transfer, (doi:10.2514/1.T5741)

There may be differences between this version and the published version. You are advised to consult the publisher's version if you wish to cite from it.

http://eprints.gla.ac.uk/189345/

Deposited on: 1 July 2019

Enlighten - Research publications by members of the University of Glasgow http://eprints.gla.ac.uk 


\title{
Comparison of Discrete Velocity Method and Gas-Kinetic Method for Binary Gas Mixtures
}

\author{
B. N. Todorova* and R. Steijl ${ }^{\dagger}$ \\ School of Engineering, University of Glasgow, University Avenue, Glasgow G12 8QQ, UK
}

The formulation of computationally efficient methods describing gas mixtures at kinetic level suitable for demanding aerospace applications presents significant challenges. In this work, we contribute a gas-kinetic scheme for binary gas mixtures in which the kinetic model is capable of recovering, in the continuum limit, the correct heat transfer, mixture viscosity as well as species diffusion. The model accounts for separate species-mean velocity such that the species diffusion and velocity drift are accurately represented. The main goal is to derive a numerically efficient GKS method which has the ability to accurately model species diffusion and velocity drift, such that two-species Navier-Stokes equations are recovered with the correct Prandtl number. The paper compares the solutions of the underlying kinetic model obtained using the GKS method and the discrete-velocity method (DVM). The limitations of the GKS for different flows and different levels of thermodynamic non-equilibrium are examined. Supersonic flows with varying species mass ratios, concentrations and Knudsen number are investigated. For the cases considered a good agreement is observed, showing that the developed GKS method provides a valuable approach for modeling these challenging flows. Also, the reduction in required CPU time for the GKS relative to DVM is shown to be significant.

\section{Nomenclature}

Latin Symbols:

$\begin{array}{ll}a & =\text { spatial derivative of the Maxwellian distribution function } \\ A & =\text { temporal derivative of the Maxwellian distribution function } \\ A_{12}^{*} & =\text { non-dimensional coefficient } \\ d_{1} & =\text { diameter of the atom taken as a sphere } \\ f & =\text { gas distribution function } \\ f^{M} & =\text { Maxwellian gas distribution function } \\ f_{s}^{0} & =\text { initial non-equilibrium gas distribution function }\end{array}$

\footnotetext{
*Ph.D. Candidate, School of Engineering, University of Glasgow. E-mail: b.todorova.1 @ research.gla.ac.uk

†Senior Lecturer, School of Engineering, University of Glasgow. E-mail: rene.steijl@ glasgow.ac.uk
} 


$$
\begin{aligned}
& f_{s}^{e q} \quad=\text { time-dependent equilibrium gas distribution function } \\
& f_{s}\left(x_{j+\frac{1}{2}}, t, u_{i}\right)=\text { time-dependent gas distribution function at cell face } \\
& \mathbf{F}_{j+\frac{1}{2}}=\text { numerical flux of macroscopic variables at cell face } \\
& g \quad=\text { equilibrium gas distribution function } \\
& g^{s h} \quad=\quad \text { Shakhov equilibrium gas distribution function } \\
& G_{s} \quad=\text { Modified Maxwellian distribution function per species } s \\
& G_{s}^{s h}=\text { Shakhov Modified distribution function per species } s \\
& k \quad=\text { Boltzmann's constant } \\
& \text { Kn } \quad=\text { local Knudsen number } \\
& \text { L } \quad=\text { characteristic length } \\
& m \quad=\text { molecular mass } \\
& m_{\text {mix }}=\text { mass of the gas mixture } m_{\text {mix }}=\rho / n \\
& m_{2} / m_{1} \quad=\text { mass ratio between species } 2 \text { and } 1 \\
& M \quad=\quad \text { free stream Mach number } \\
& n \quad=\text { gas number density } \\
& n_{s} \quad=\text { species number density } \\
& n_{1} / n=\text { concentration of light species in the gas mixture } \\
& p \quad=\text { pressure } \\
& \operatorname{Pr} \quad=\text { Prandtl number } \\
& q_{x} \quad=\text { heat flux in the } \mathrm{x} \text {-direction } \\
& q_{x_{s}}^{\text {corr }} \quad=\text { species heat flux correction } \\
& Q_{s} \quad=\text { collision term per species } s \\
& S_{S} \quad=\text { species source term in continuum flow } \\
& t \quad=\text { time } \\
& T \quad=\text { gas temperature } \\
& \hat{T} \quad=\text { modified gas temperature } \\
& T_{\text {ref }} \quad=\text { reference gas temperature } \\
& u, v, w=\text { molecular velocity components } \\
& \mathbf{u}_{0} \quad=\text { mean gas velocity } \\
& \mathbf{u}_{s} \quad=\text { species mean velocity } \\
& \mathbf{u}_{s}^{(g)} \quad=\text { modified species velocity } \\
& \mathbf{W}_{j}=\text { conserved macroscopic variables }
\end{aligned}
$$




\section{Greek Symbols}

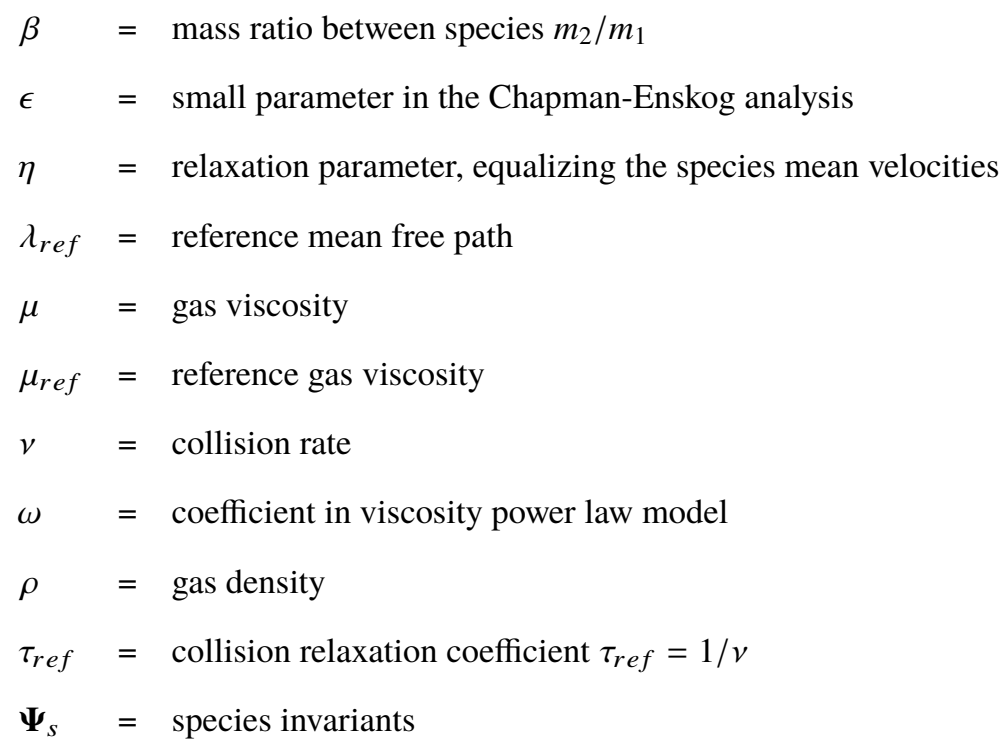

\section{Subscripts and superscripts}

()$_{s} \quad=$ species variables

()$_{j} \quad=$ cell

()$^{n}=$ time step

\section{I.Introduction}

The flowfield around a hypersonic vehicle contains both continuum and rarefied regions with strong gradients of the macroscopic variables. In addition, thermal and chemical non-equilibrium processes can occur, depending on the conditions encountered. The thermodynamic and thermo-chemical non-equilibrium processes require a multiple-species formulation for the gas. Examples of flows where these phenomena occur include strong shocks causing variable species concentrations even in flows without chemical reactions, by effectively separating within the shock the heavier from the lighter species in the gas mixture. Furthermore, dissociation and recombination reactions will occur in many high-enthalpy applications, leading to gradients in species concentrations and therefore a need to model species diffusion accurately. Such multi-scale problems require a detailed description of the physics, e.g. governed at the level of kinetic theory. The general governing equation for kinetic modeling for gas flows is the Boltzmann equation[1]. Because of the complexity and computational overhead involved in solving this equation, mainly as a result of the complex particle collision term, a number of well-known model equations have been developed, e.g. BGK [2], Shakhov [3], ES [4]. In rarefied gas dynamic simulations, the Direct Simulation Monte Carlo (DSMC) method [5] is widely used and statistically approximates solutions to the Boltzmann equation. An alternative approach involves deterministic 
methods based on discretization of the phase space. These Discrete Velocity Methods (DVM) [6] require significant computational resources and for the vast majority of aerospace applications involving hypersonic and rarefied flows are not feasible. The DSMC method, however, loses efficiency and potentially also accuracy for reduced mean speeds in the considered flow as well as for increasing densities. Therefore, for flows in the transitional regime between rarefied and continuum, particular challenges exist for CFD methods.

The present work considers gas-kinetic methods for the simulation of binary mixtures of monoatomic gases in the continuum as well as moderately rarefied conditions. The main aim was to obtain sufficient physical detail and accuracy, while achieving computational efficiency, enabling engineering simulations of realistic aerospace applications, including hypersonic, high-altitude flight of hypersonic cruise vehicles and spacecraft. This work builds on previous work by the authors in developing GKS methods for single-species diatomic gas flows[7] and forms a first stepping stone to a GKS method for (chemically-reacting) diatomic gas mixtures.

The gas-kinetic BGK scheme was introduced by $\mathrm{Xu}$ and Prendergast [8, 9] and provides an alternative solution methodology as compared to more conventional approximate Riemann-solver based finite-volume CFD methods. The main difference is in the flux computation, which is obtained from the integration of the time-dependent gas distribution function at a cell face. This gas distribution function follows from a cell-face local kinetic problem constructed from local continuum flow variables. The GKS is based on BGK models and recovers the Navier-Stokes equations in the continuum limit. As originally introduced, GKS methods were Navier-Stokes solvers, and more recently the GKS approach has been extended by a number of authors, e.g. [7, 10,11], with a capability to resolve thermal non-equilibrium and flow rarefaction effects. The strength of the gas-kinetic scheme lies in its capability to include the effect of collisions introducing a multi-scale effect which is missing in more commonly-used Navier-Stokes schemes. Moreover, due to their nature, gas-kinetic schemes can predict flow fields with a certain degree of rarefaction, enabling the computational analysis of a wide range of high-speed flows from the continuum to the rarefied regime without having to rely on different numerical methods. For simulation of flows covering a wide range of rarefaction, $\mathrm{Xu}$ and co-workers introduced the UGKS method [12, 13], more recently extended to diatomic gas flows with rotational relaxation[14] and vibrational and rotational relaxation[15]. The computational overhead of the UGKS is however similar to that of a DVM approach and therefore, despite the capabilities of the UGKS methodology, further work in the development of more computationally efficient methods is still an active line of research, e.g. [7, 16, 17].

Xu introduced a multicomponent gas-kinetic scheme [18] based on an inviscid gas-kinetic BGK scheme with common mean velocity and temperature. This model was extended to a chemically reacting mixture [19] and more recently to a fourth order multi-species GKS [20] in order to overcome the numerical dissipation caused by the lower order method. A unified gas-kinetic scheme [21] has been constructed for a gas mixture, based on the single-BGK-operator model[22], capable of recovering one transport coefficient correctly. Wang and Xu [21] validate the scheme by inspecting a shock structure under different conditions and the micro channel problem and suggest that some of the discrepancies in the 
results are due to the selected mixture kinetic model. Most recently a discrete unified gas kinetic scheme (DUGKS) for a binary mixture [23] has been developed, again based on the single-BGK-operator model [22]. The DUGKS possesses all the advantages and shows excellent agreement with the UGKS, while being computationally more efficient. As outlined in that paper, a limitation in both UGKS and DUGKS is connected with the chosen kinetic model that has only one correct transport coefficient in the continuum limit. Very recently the McCormack method was applied with the DUGKS [24] in order to overcome this disadvantage. All transport coefficients are correctly fitted, but the method itself introduces a restriction on the level of non-equilibrium in the flow. Clearly, there is a need for further improvements of the kinetic models, as well as the numerical schemes for gas mixtures.

In the present work, the limitations of these previous works are overcome with a novel kinetic model which accurately represents (qualitatively as well as quantitatively) the mixture viscosity, heat transfer as well as species diffusion [25, 26]. In this previous work the kinetic model is shown to have the correct thermal conductivity expression and Prandtl number for the gas mixture [25]. In [26] the model is numerically tested and validated with results from the full Boltzmann equation [27] and DSMC [28]. A key benefit of the model is the corrected thermal conductivity in the heat flux and in [26] this is numerically demonstrated. The heat flux of the gas mixture matches closely with the results for the heat flux of the Boltzmann equation. The key contribution of the present work is a new GKS method based on this kinetic model with an important advantage over previous mixture GKS methods. First, the current method employs separate species-mean velocities such that species diffusion and velocity drift are accurately represented. Secondly, the GKS method presented here has the correct transport properties in the continuum limit, including the recovery of the correct Prandtl number. This GKS method captures detailed level of physics in non-equilibrium flows that normally require more detailed and computationally demanding approaches, e.g. DSMC or discrete-velocity methods (DVM) for moderate levels of rarefaction of the flow. A key aspect of the GKS approach is the computational efficiency together with the level of non-equilibrium in mixture flows that can be accurately resolved. The second part of this paper addresses this question using a detailed comparison between the new GKS and DVM solutions based on the new kinetic model.

The paper begins with the discrete velocity method and the gas kinetic scheme procedures and outlines the main differences between the two solution processes. A brief review of kinetic theory, the Boltzmann and model equations, followed by a discussion of the recently introduced kinetic model [25] follows in section III. Then, the GKS method development and its formulation are introduced in section IV. For the binary mixture kinetic model, a number of sample solutions, obtained using the discrete velocity method (DVM) and the new GKS methods are presented in section V. A detailed comparison is shown where the DVM results represent a a benchmark for the GKS. The complex flow physics occurring in a normal shock in a binary mixture is considered to highlight the species diffusion and velocity drift effects and their dependencies on the species atomic mass ratios and concentration. A two-dimensional flow over a flat plate is also studied for different flow conditions, where strong non-equilibrium effects of interest occur particularly around 
the leading edge of the plate. The key advantage of the GKS is the computational efficiency of the scheme, which is discussed in detail for the presented baseline test cases. Finally, Section VI. summarizes key findings and future work.

\section{DVM vs GKS}

In this section the procedure of the discrete velocity method (DVM) and the gas kinetic scheme (GKS) are summarized. Figures 1 (a) and (b) demonstrate how each of the schemes is formulated for a one dimensional flow. In the DVM approach used here, the phase(velocity)-space is discretized using a uniform Cartesian mesh. In each cell of the space mesh, this velocity-space is used to define discretized non-equilibrium and equilibrium distribution functions. For each of these velocities, the numerical flux of the non-equilibrium distribution function through each cell face is computed to represent the convection part of the kinetic Boltzmann equation. The discretized non-equilibrium and equilibrium distribution functions in each cell center are used to evaluate the collision term based on the BGK approximation. In each time step, the convection and collision effects create a modified non-equilibrium distribution function, from which the corresponding continuum quantities are obtain from taking moments in velocity space. Finally, an updated equilibrium distribution function is created based on these continuum quantities in each cell center. Both the number of velocities and the bounds of the velocity space depend on the problem and increase drastically for 2D and 3D problems. The process of the gas kinetic scheme is completely different. The main idea of the gas kinetic scheme is to avoid the storage of the non-equilibrium and equilibrium distribution function in each cell center as well as the need to take numerical moments of discretized non-equilibrium distribution functions. Instead, updates of the continuum quantities in each cell center are obtained directly by a finite-volume scheme using fluxes of the conserved variables. The evaluation of fluxes consists of constructing the distribution function from the macroscopic variables and its gradients, expressing the flux of the function with a Chapman-Enskog expansion and then taking moments to receive the analytical expression for the flux of conserved variables. In Fig 1 b), the box drawn around the considered cell face represents this reconstructed kinetic solution. The moments of the locally-constructed kinetic solution are evaluated analytically. This means that this kinetic solution is not actually stored and that the flux evaluation consists in evaluating the analytical solutions derived for these moments. The GKS solver resembles a continuum solver in its structure and also in its computational expense.

Since the gas-kinetic scheme is based on the first-order in Knudsen number Chapman-Enskog expansion, only small to moderate levels of rarefication can be modeled. This limitation will be explored in the numerical section. The first order expansion also means that the current scheme reduces to the Navier-Stokes equations in the hydrodynamic limit. At the same time the scheme allows to model beyond the capabilities of a continuum solver in regions of strong thermodynamic non-equilibrium and provides a method to connect microscopic behavior to macroscopic quantities. 


\section{DVM}

GKS

$(\underline{\varrho}, \underline{\varrho} \underline{u}, \underline{\varrho e})_{(i-1)} \quad(\underline{\varrho}, \underline{\varrho} \underline{u}, \underline{\varrho} e)_{(i)}$

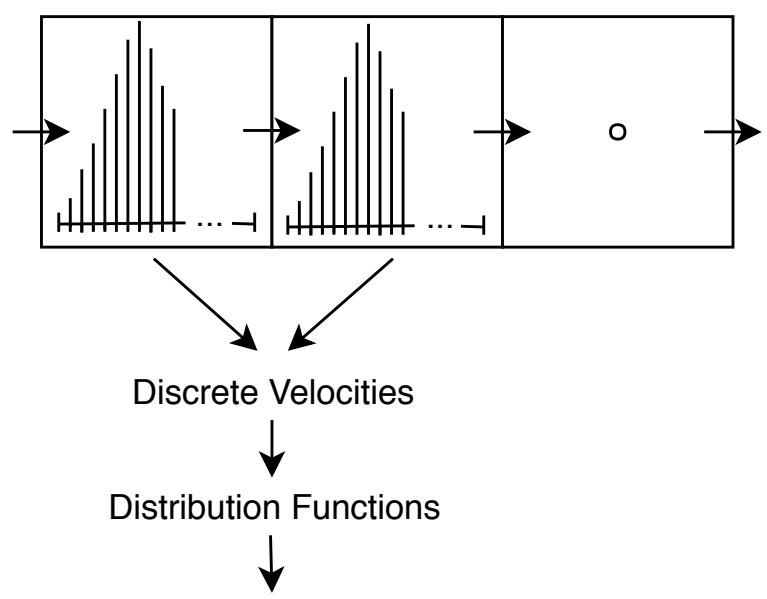

Flux Fuctions

(a) Discrete Velocity Method

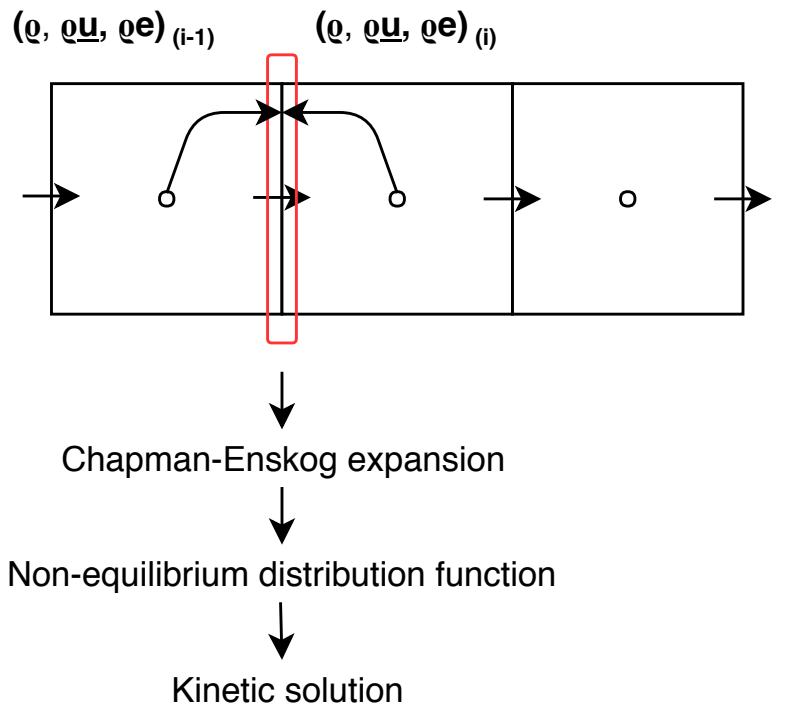

(b) Gas kinetic scheme

Fig. 1 Discrete Velocity and Gas Kinetic Scheme Diagrams

\section{Kinetic Model}

The Boltzmann transport equation is a fundamental equation of molecular dynamics and is capable of modelling gas flows and mixtures with different rarefaction levels- from continuum to free molecular regime. It examines the rate of change of the distribution function with respect to time and position by accounting for the collision processes that take place [29]. Crucial properties of the Boltzmann equation include the conservation of properties like mass, momentum and energy during collisions, entropy dissipation and collision equilibria. However, the computational cost associated with a direct discretization and solutions of the Boltzmann equation make this approach impossible to apply for the vast majority of practical problems in aerospace engineering. Instead, relaxation model equations, simplifying the collision integral have been introduced.

Many practical applications in high-speed and rarefied gas dynamics require the evaluation of flow comprised of more than one gas. The extension of a BGK-style relaxation model to a mixture of gases is a challenging task and the well-known disadvantage of recovering a unit Prandtl number (while $\operatorname{Pr}=2 / 3$ for monoatomic gas) is still present. Viscosity, heat conduction and mass diffusion coefficients are three of the transport coefficients in a multi-species gas. Single relaxation BGK models [22] can recover only one correctly. Groppi, Monica and Spiga's [30] drift velocity relaxation term facilitates fitting two transport coefficients. We have extended that model with a Shakhov-type [3] correction and managed to recover the qualitative, but also quantitative values of three transport coefficients. The 
correct hydrodynamic limit is recovered in the new Shakhov-based mixture kinetic model, which is detailed in [25] and summarized in this section. In the following, a quasi one-dimensional flow is considered, with molecular motions in all three coordinate directions, and with a macroscopic gas motion and gradients only in the $x$-direction. For a binary gas mixture the governing equation is per species $s=1,2$ (Eq. (1)) and the simplified collision operator $Q_{s}$ involves the Shakhov-based mixture distribution function $G_{s}^{s h}$ and a common collision rate $v$.

$$
\frac{\partial f_{s}}{\partial t}+u \frac{\partial f_{s}}{\partial x}=Q_{s} \quad ; \quad Q_{s}=v\left(G_{s}^{s h}-f_{s}\right) ; \quad ; \quad s=1,2
$$

where the common collision rate is defined by the gas mixture properties: the pressure $p$ and viscosity $\mu$ based on the mixture temperature $T$, such that $v=p / \mu$. In the numerical simulations the power-law viscosity is used and the molecular potential is defined by the value of $\omega$ in $\mu=\mu_{\text {ref }}\left(\frac{T}{T_{r e f}}\right)^{\omega}$. The reference viscosity $\mu_{r e f}$ is taken for a smooth, rigid, elastic sphere [1] with diameter $d_{1}$ and mass of the mixture $m_{m i x}=\rho / n$ as:

$$
\mu_{\text {ref }}=\frac{5}{16 d_{1}^{2}} \sqrt{\frac{k m_{\text {mix }} T}{\pi}},
$$

while $T_{\text {ref }}$ is the initial gas mixture temperature. The same refence values and non-dimenionalization procedure is followed as in [25].

From the species macroscopic variables, the overall gas mixture properties are obtained as shown in Eq. (3) and Eq. 4):

$$
\begin{gathered}
\sum_{s=1}^{2} n_{s}=n ; \quad \sum_{s=1}^{2} \rho_{s}=\rho ; \sum_{s=1}^{2} \rho_{s} u_{s}=\rho_{0} u_{0} \quad ; \quad \sum_{s=1}^{2} \rho_{s}\left(u_{s}-u_{0}\right)^{2}=\sum_{s=1}^{2} \rho_{s}\left|u_{s}\right|^{2}-\rho\left|u_{0}\right|^{2} \\
\frac{3}{2} n k T=\sum_{s=1}^{2} \frac{3}{2} n_{s} k T_{s}+\frac{1}{2} \sum_{s=1}^{2} \rho_{s}\left(\underline{u}_{s}-\underline{u}_{0}\right)^{2} .
\end{gathered}
$$

The target equilibrium distribution function in the collision term is defined as:

$$
\begin{aligned}
G_{s}^{s h}= & n_{s}\left(\frac{m_{s}}{2 \pi k \hat{T}}\right)^{3 / 2} \exp \left[-\frac{m_{s}}{2 k \hat{T}}\left(\left(u-u_{s}^{(g)}\right)^{2}+v^{2}+w^{2}\right)\right] * \\
& \left\{1+\frac{2(1-\operatorname{Pr})\left(u-u_{s}^{(g)}\right) q_{x_{s}}^{c o r r}}{5 p_{s} k \hat{T} / m_{s}}\left[\frac{m_{s}}{2 k \hat{T}}\left(\left(u-u_{s}^{(g)}\right)+v^{2}+w^{2}\right)-\frac{5}{2}\right]\right\},
\end{aligned}
$$

with species pressure $p_{s}$ defined as, $p_{s}=\rho_{s} k \hat{T} / m_{s}=n_{s} k \hat{T}$, and a heat flux correction $q_{x_{s}}^{\text {corr }}$, integrated with the species 
velocities $u_{s}$ :

$$
\begin{gathered}
\int_{-\infty}^{\infty} m_{s}\left(u-u_{s}\right) \frac{1}{2}\left(\left(u-u_{s}\right)^{2}+v^{2}+w^{2}\right)\left\{G_{s}^{s h}-\frac{\epsilon}{v}\left(\frac{\partial f_{s}^{M}}{\partial t}+u \frac{\partial f_{s}^{M}}{\partial x}\right)\right\} d \underline{u}=-\frac{\epsilon}{v} \frac{5}{2} k \frac{n_{s} k T}{m_{s}} \frac{\partial T}{\partial x}+(1-\operatorname{Pr}) q_{s}^{c o r r}+O\left(\epsilon^{2}\right), \\
q_{x_{s}}^{\text {corr }}=-\frac{\epsilon}{v} \frac{5}{2} \frac{k}{\operatorname{Pr}} \frac{n_{s} k T}{m_{s}} \frac{\partial T}{\partial x}+O\left(\epsilon^{2}\right)
\end{gathered}
$$

where $\epsilon$ is the small parameter (related to the Knudsen number) used in the Chapman-Enskog (CE)[1] analysis. A modified species mean velocity $u_{s}^{(g)}$ is introduced that differs from the local species mean velocity as well as mixture velocity. Furthermore, a modified temperature $\hat{T}$ is introduced, common for both species, and different from the mixture temperature $T$. Based on these modified gas properties used in the equilibrium distribution function, the model can recover the correct species diffusion, viscosity and heat conduction coefficients in the continuum limit. Introducing the collision terms, evaluating the integrals and combining with the moment conservation equation, the species velocity in the distribution function $G_{s}^{s h}$ is expressed from local species velocity and the average gas mixture velocity:

$$
u_{s}^{(g)}=\left(1-\frac{\eta}{v}\right) u_{s}+\frac{\eta}{v} u_{0} .
$$

From the energy conservation, the modified temperature is in the form:

$$
\hat{T}=T-\frac{1}{3 n k} \sum_{s=1}^{2} \rho_{s}\left(u_{s}^{(g)}-u_{0}\right)^{2}=T-\frac{1}{3 n k}\left(1-\frac{\eta}{v}\right)^{2} \sum_{s=1}^{2} \rho_{s}\left(u_{s}-u_{0}\right)^{2} .
$$

The expressions for $u_{s}^{(g)}$ and $\hat{T}$ depend on the macroscopic velocities and temperatures of the species and the gas mixture, but also on the relaxation parameters $v$ and $\eta$. The $v=p / \mu$ parameter is related to the governing equation (Eq. (1)) and $\eta$ is introduced by Groppi et al. [30] as a constraint on the species velocity equalization:

$$
\frac{1}{n_{1}} \iiint_{-\infty}^{\infty} \underline{u} Q_{1} d \underline{u}-\frac{1}{n_{2}} \iiint_{-\infty}^{\infty} \int_{\underline{u}} Q_{2} d \underline{u}=-\eta\left(\underline{u}_{1}-\underline{u}_{2}\right) .
$$

In practical applications the two relaxation coefficients $v$ and $\eta$ are related to the viscosity and diffusion coefficients respectively. The derivation of the $\eta / v$ expression is shown in [25] and results in:

$$
\frac{\eta}{v}=\frac{5}{3} \frac{1}{\left(m_{1}+m_{2}\right) A_{12}^{*}} \frac{\rho}{n}
$$

where $A_{12}^{*}$ is a non-dimensional coefficient and for noble mixtures a good approximation for $A_{12}^{*}$ is $A_{12}^{*}=1.11$ [31].

Note that the indifferentiability principle, described by Garzo et al. [32], requires the Shakhov-based model for species to reduce to a single-species Shakhov model when the species are identical. This means that for the same 
masses $\left(m_{1}=m_{2}\right)$ the sum of the distribution function $G_{s}^{S h}$ should lead to a distribution function $G$ that satisfies the single-species Shakhov model [3], where $G=\sum_{s=1}^{2} G_{s}^{S h}$. A necessary assumption [33] is that $u_{s}=u_{0}$ for all species s. It follows from Eq. (8) and Eq. (9) that $u_{s}=u_{0}=u_{s}^{(g)}$ and $T=\hat{T}$. Under these conditions the sum over $s$ for the distribution function $G_{s}^{S h}$ in Eq. (6) leads to a single-species distribuion function $G\left(n, m, u_{0}, T\right)$. This means that for the same properties of the two species the binary-mixture model satisfies the indifferentiability principle and recoves the single-species Shakhov-model. Note also that numerically for $m_{1}=m_{2}$ there is no mechanism to separate the two species from each other under the same initial conditions.

Based on the described novel kinetic model, a gas kinetic scheme is developed.

\section{Development of a Gas-kinetic Scheme}

\section{A. Governing Equations}

In this section, the gas-kinetic scheme for a well-resolved flow is presented, based on the newly developed mixture model [25] and the original gas-kinetic BGK scheme [8-10]. It is important to note that unlike a single species gas, momentum and energy are conserved only for the gas mixture. This means that the species momentum equations will return a source term $S_{s}$. The source term is created due to the difference between species velocities, which is of order $O(\epsilon)$ and therefore disappears, if the gas-kinetic scheme reduces to the equivalent Euler equations. However, the presented GKS is derived for viscous flow and therefore $O(\epsilon)$ terms are included. Separate species momentum equations are used which include source as defined previously. The species conservation, species momentum and total energy continuum equations are in the form (Eq. 12)-(14)):

$$
\begin{gathered}
\frac{\partial n_{s}}{\partial t}+\frac{\partial}{\partial x}\left\{n_{s} u_{0}-\epsilon \frac{k T}{\eta}\left[\frac{1}{m_{s}} \frac{\partial n_{s}}{\partial x}-\frac{n_{s}}{\rho} \frac{\partial n}{\partial x}+\frac{n_{s}}{T}\left(\frac{1}{m_{s}}-\frac{n}{\rho}\right) \frac{\partial T}{\partial x}\right]\right\}=0 \\
\frac{\partial\left(\rho_{s} u_{s}\right)}{\partial t}+\frac{\partial}{\partial x}\left[n_{s} k \hat{T}+\rho_{s} u_{0}^{2}-2 \rho_{s} u_{0}\left(u_{0}-u_{s}\right)+\left(1-\frac{\eta}{v}\right)^{2}\left(u_{0}-u_{s}\right)^{2}-\mu\left(\frac{4}{3} \frac{\partial u_{0}}{\partial x}\right)\right]=v \rho_{s}\left(\frac{\eta}{v}\right)\left(u_{0}-u_{s}\right),
\end{gathered}
$$

with viscosity coefficient $\mu=(\epsilon / v) n k T$. As expected, the right-hand side of Eq. (13) results in a source term $S_{s}$ for each species. The summation of the source terms over all species goes to zero, which confirms that the system is conservative for the mixture. The energy equation for the gas mixture is in the form:

$$
\begin{array}{r}
\frac{\partial}{\partial t}\left[\frac{3}{2} n k T+\frac{1}{2} \rho u_{0}^{2}\right]+\frac{\partial}{\partial x}\left\{\sum_{s=1}^{2} m_{s} n_{s}\left[\frac{5}{2} \frac{k T}{m_{s}} u_{0}+\frac{1}{2} u_{0}^{3}+\left\{\frac{5}{2} \frac{k T}{m_{s}}+\frac{3}{2} u_{0}^{2}\right\}\left(u_{0}-u_{s}\right)\right]\right. \\
\left.-\epsilon \frac{k T}{v} \sum_{s=1}^{2}\left[\frac{4}{3} n_{s} u_{0} \frac{\partial u_{0}}{\partial x}+\frac{1}{\operatorname{Pr}} \frac{5}{2} n_{s}\left(\frac{k}{m_{s}}\right) \frac{\partial T}{\partial x}\right]\right\}=0
\end{array}
$$


In Eq. [12, the second term within curly brackets represents the velocity drift between species mean velocity $u_{s}$ and the mixture mean velocity $u_{0}$, caused by diffusion in case of different molecular masses of the species.

\section{B. Cell Face Distribution Function}

The mathematical development of the GKS concerns with the evolution of the species distribution function at a cell face. In the following, a quasi-1D flow is considered. The numerical fluxes for a cell face $(j+1 / 2)$ between cell $j$ (left) and cell $j+1$ (right) can be obtained from the time dependent distribution function (Eq. 15) around the interface $x_{j+1 / 2}$.

$$
f_{s}\left(x_{j+1 / 2}, t, u\right)=v \int_{0}^{t} f_{s}^{e q}\left(x^{\prime}, t^{\prime}, u\right) \exp \left(-v\left(t-t^{\prime}\right)\right) d t^{\prime}+\exp (-v t) f_{s}^{0}\left(x_{j+1 / 2}-u t, 0, u\right)
$$

with $x^{\prime}=x_{j+1 / 2}-u\left(t-t^{\prime}\right)$ - the particle trajectory during the time-step considered and $u$ is the particle velocity in the x-direction. A characteristic of the GKS method is that the inviscid and viscous fluxes are obtained simultaneously, since the distribution function comprises of equilibrium and non-equilibrium contributions. The initial distribution function $f_{s}^{0}$ around the interface is based on a CE expansion as

$$
f_{s}^{0}(x, 0, u)=G_{s}^{s h}(x, 0)-\frac{1}{v}\left(\frac{\partial f_{s}^{M}}{\partial t}+u \frac{\partial f_{s}^{M}}{\partial x}\right)
$$

where $G_{s}^{s h}(x, 0)$ is the modified Maxwellian in the mixture kinetic model evaluated for the conditions in position $x$ at time $t=0$, while $f_{s}^{M}$ are the unmodified Maxwellian distribution function. Assuming a linear variation of the distribution function within the cells, the expression is expanded as:

$$
\begin{aligned}
f_{s}^{0}(x, 0, u)= & G_{s}^{s h}\left(x_{j+1 / 2}, 0\right)+\left.\frac{\partial G_{s}^{s h}}{\partial x}\right|_{x_{j+1} / 2}\left(x-x_{j+1 / 2}\right) \\
& -\left.\frac{1}{v}\left(\frac{\partial f_{s}^{M}}{\partial t}+u \frac{\partial f_{s}^{M}}{\partial x}\right)\right|_{x_{j+1 / 2}}-\left.\frac{1}{v}\left(\frac{\partial^{2} f_{s}^{M}}{\partial t \partial x}+u \frac{\partial^{2} f_{s}^{M}}{\partial x^{2}}\right)\right|_{x_{j+1 / 2}}\left(x-x_{j+1 / 2}\right), \\
f_{s}^{0}(x, 0, u) \approx & G_{s}^{s h}\left(x_{j+1 / 2}, 0\right)+f_{s}^{M}\left[a\left(x-x_{j+1 / 2}\right)-\frac{1}{v}(u a+A)\right],
\end{aligned}
$$

where $a$ and $A$ represent spatial and temporal derivatives as:

$$
a=\frac{1}{f_{s}^{M}} \frac{\partial f_{s}^{M}}{\partial x} \quad ; \quad \frac{\partial f_{s}^{M}}{\partial x} \approx \frac{\partial G_{s}^{s h}}{\partial x} ; A=\frac{1}{f_{s}^{M}} \frac{\partial f_{s}^{M}}{\partial t}
$$

where the derivatives are evaluated at $x_{j+1 / 2}$ and the assumption is made that the derivative of $G_{s}^{s h}$ can be approximated by the derivative of $f_{s}^{M}$ for the order of accuracy in $v$ and space considered. The equilibrium distribution $f_{s}^{e q}$ around 
the cell interface is also approximated using a second-order accurate Taylor series expansion:

$$
f_{s}^{e q}(x, t, u)=G_{s}^{s h}\left(x_{j+1 / 2}, 0\right)+f_{s}^{M}\left[a\left(x-x_{j+1 / 2}\right)+A t\right] .
$$

Unlike the initial non-equilibrium function $f_{s}^{0}$, which is independent in time, the equilibrium function varies within a time-step due to the fact that particle collisions are considered along with particle convection. Using Eq. 19], the integral on the right-hand side of Eq. (15) becomes:

$$
\begin{aligned}
v \int_{0}^{t} f_{s}^{e q}\left(x^{\prime}, t^{\prime}, u\right) \exp \left(-v\left(t-t^{\prime}\right)\right) d t^{\prime} & =v G_{s}^{s h}\left(x_{j+1 / 2}, 0\right) \int_{0}^{t} \exp \left(-v\left(t-t^{\prime}\right)\right) d t^{\prime} \\
& +v f_{s}^{M} \int_{0}^{t}\left[-u a\left(t-t^{\prime}\right)+A t^{\prime}\right] \exp \left(-v\left(t-t^{\prime}\right)\right) d t^{\prime} \\
= & {[1-\exp (-v t)] G_{s}^{s h}\left(x_{j+1 / 2}, 0\right)+f_{s}^{M}\left[-a u \frac{1}{v}+a u\left(t+\frac{1}{v}\right) \exp (-v t)+A t-\frac{1}{v}[1-\exp (-v t)] A\right] . }
\end{aligned}
$$

Using Eq. [17), the second term on the right-hand side of Eq. [15] becomes:

$$
\exp (-v t) f_{s}^{0}\left(x_{j+1 / 2}, 0, u\right)=G_{s}^{s h}\left(x_{j+1 / 2}, 0\right) \exp (-v t)+f_{s}^{M}\left[-a u t-\frac{1}{v}(u a+A)\right] \exp (-v t)
$$

Combining Eq. 201 and (21), for a well-revolved flow, Eq. (15) becomes the time-dependent gas distribution function on the cell face, from which the flux of macroscopic variables can be expressed:

$$
f_{s}\left(x_{j+1 / 2}, t, u\right)=G_{s}^{s h}\left(x_{j+1 / 2}, 0\right)-\left[\frac{1}{v}(a u+A)-A t\right] f_{s}^{M}=G_{s}^{s h}\left(x_{j+1 / 2}, 0\right)-\frac{1}{v}\left(\frac{\partial f_{s}^{M}}{\partial t}+u \frac{\partial f_{s}^{M}}{\partial x}\right)+t \frac{\partial f_{s}^{M}}{\partial t}
$$

The time-dependent numerical fluxes across the cell face are evaluated by taking moments of the time-dependent distribution function on the cell face:

$$
\mathbf{F}_{j+\frac{1}{2}}=\sum_{s=1}^{2} \int u \boldsymbol{\Psi}_{s} f_{s}\left(x_{j+1 / 2}, t, u\right) d \underline{u},
$$

where $\boldsymbol{\Psi}_{1}=\left(1,0, m_{1} u, 0, \frac{1}{2} m_{1}\left(u^{2}+v^{2}+w^{2}\right)\right)^{T}$ and $\boldsymbol{\Psi}_{2}=\left(0,1,0, m_{2} u, \frac{1}{2} m_{2}\left(u^{2}+v^{2}+w^{2}\right)\right)^{T}$.

The conserved macroscopic variables $\mathbf{W}_{\mathbf{j}}$ (Eq. (24)) are the variables the GKS method stores in the cell centers and 
which are integrated in time:

$$
\mathbf{W}_{j}=\left(\begin{array}{c}
n_{1} \\
n_{2} \\
\rho u_{1} \\
\rho u_{2} \\
\frac{3}{2} n k T+\frac{1}{2} \rho u_{0}^{2}
\end{array}\right)_{j}
$$

The update of the conservative variables from time step $n$ to $(n+1)$ is then defined as:

$$
\mathbf{W}_{\mathbf{j}}^{\mathbf{n}+\mathbf{1}}=\mathbf{W}_{\mathbf{j}}^{\mathbf{n}}+\frac{1}{\Delta x} \int_{t^{n}}^{t^{n+1}}\left(\mathbf{F}_{\mathbf{j}-\mathbf{1} / \mathbf{2}}(t)-\mathbf{F}_{\mathbf{j}+\mathbf{1} / \mathbf{2}}(t)\right) d t+\Delta t\left(0,0, S_{1}, S_{2}, 0\right)^{T}
$$

where the source term is defined as shown in Eq. 26:

$$
S_{s}=v \rho_{s}\left(\frac{\eta}{v}\right)\left(u_{0}-u_{s}\right) ; s=1,2
$$

The GKS model described above for quasi one-dimensional flows was further derived for two-dimensional flows on a curvilinear mesh. In the two-dimensional formulation, a direction-cosine approach is followed and the cell-face normal direction replaces the $x$-direction in the discussion above for 1D flows. The velocity-space integrals were worked out analytically to provide the main efficiency gain of a GKS approach relative to the DVM method.

\section{Numerical Results}

The newly developed GKS scheme is tested in this section and compared to DVM solutions for the same kinetic model. Two different test cases, e.g. the flow through a normal shock wave and the rarefied supersonic flow over a flat plate, are considered. The normal shock test case represents the simplest problem that involves strong gradients and non-equilibrium flow and is also well documented. The focus is on the effect of treating the gas as a mixture, inspecting the diffusion effect and species properties. The change in the flow is demonstrated for different Knudsen numbers and species mass ratios. The kinetic model is implemented with the discrete velocity method (DVM) and the gas kinetic scheme (GKS). DVM allows for strong non-equilibrium flows, since there are no underlying assumptions for small deviations as in the CE expansion, required for the GKS. The high computational expense of the DVM is feasible for one and two-dimensional cases, but the extension to vehicles of practical application will require an alternative approach, e.g. the GKS method or a hybrid GKS/DVM approach. The solutions provided by the GKS and DVM methods are compared, based on the same kinetic model. Ideally, the results from the GKS will be as close as possible to the results of the DVM. The computational cost of both numerical approaches is also detailed. 


\section{A. Shock Structure}

A classical problem for rarefied flows is the study of a normal shock wave for single as well as multi-species gases. Monoatomic binary mixtures have been investigated extensively, experimentally [34] [35] and numerically [27], providing a dataset for validation.

\section{Table 1 Test Case Conditions for a Normal Shock Wave}

\begin{tabular}{cccc}
\hline$M$ & $m_{2} / m_{1}$ & $n_{1} / n$ & $\omega$ \\
\hline 1.5 & 2 & 0.9 & 0.5 \\
1.5 & 2 & 0.5 & 0.5 \\
1.5 & 4 & 0.9 & 0.5 \\
1.5 & 4 & 0.9 & 0.72 \\
\hline
\end{tabular}

In this section the profile of a normal shock wave is studied for a binary mixture of gases with different molecular masses. Test case conditions are summarized in Table 1. Here $M$ is the free-stream Mach number, $m_{2} / m_{1}$ defines the heavy to light gas mass ratio, $n_{1} / n$ is the concentration of light gas in the flow. The $\omega=0.5$ refers to a hard-sphere molecular potential and $\omega=0.72$ to a variable hard-sphere potential. The goal is to analyze the effect of different free stream conditions (ratio of masses, concentration ratio and molecular potential) on the macroscopic flow variables.

Figure 2 shows the normalized number density for each species and the mixture temperature through the shock under the specified conditions, starting with free stream Mach number of 1.5 and varying the concentration (50\% and $90 \%$ light species) and mass $\left(m_{2} / m_{1}=2,4 ;\right)$ ratios (Fig 2 (a)-(d)). These flow-conditions are chosen to allow for a numerical comparison with the results from Kosuge et. al [27] for a normal shock using the Boltzmann equation, represented by the red elements. The DVM results are in blue and the GKS results are in green. It is important to stress that the model in Kosuge's work is not a single-relaxation time BGK model and therefore the DVM results for species kinetic model will also deviate from those results. The reference length of the problem is defined as:

$$
L_{r e f}=\frac{u_{r e f}}{t_{r e f}}=\frac{u_{r e f}}{\tau_{r e f}}=\frac{u_{r e f} \mu_{r e f}}{p_{r e f}},
$$

where the reference viscosity is defined as in Eq. (2) and $\tau_{r e f}=1 / v$ is a relaxation ratio coefficient. The mean free path is defined as $\lambda_{\text {ref }}=1 /\left(\sqrt{2} \pi d_{1}^{2} n\right)$ in the solution of the full Boltzmann equation [27]. We use the ratio between the reference length $L_{r e f}$ and the mean free path $\lambda_{r e f}$ as a scaling factor:

$$
\frac{L_{r e f}}{\lambda_{\text {ref }}}=\frac{5 \sqrt{\pi}}{8} \sqrt{\frac{n_{1}+\beta n_{2}}{n_{1}+n_{2}}}
$$

where the constant $\beta=m_{2} / m_{1}$ is the mass ratio. For increasing levels of thermodynamic non-equilibrium this difference in the used kinetic models will be more pronounced. A key aspect of the comparison between our DVM and GKS 


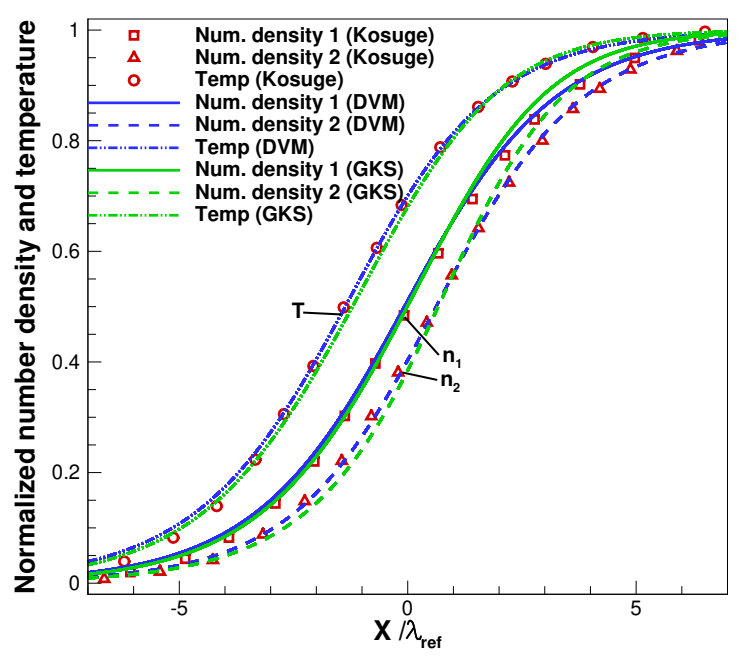

(a) $M=1.5 ; m_{2} / m_{1}=2 ; n_{1} / n=0.9$

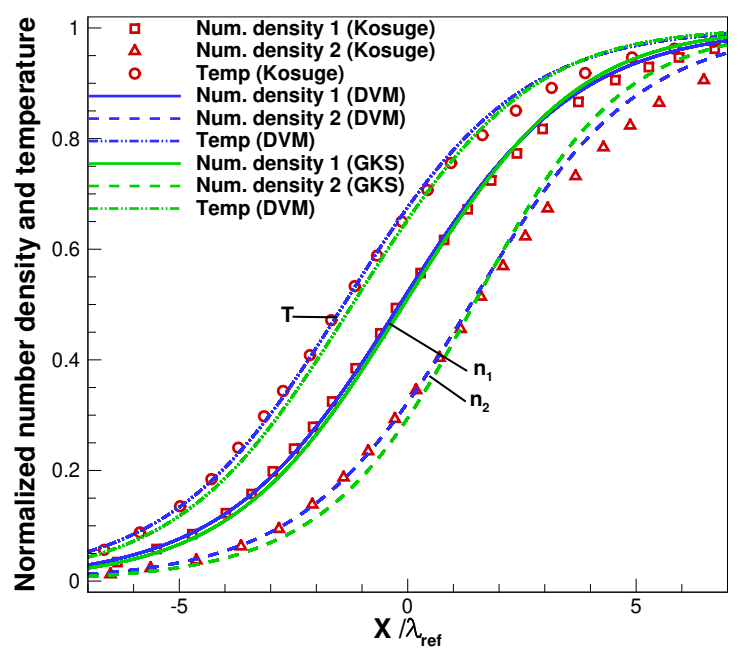

(c) $M=1.5 ; m_{2} / m_{1}=4 ; n_{1} / n=0.9$

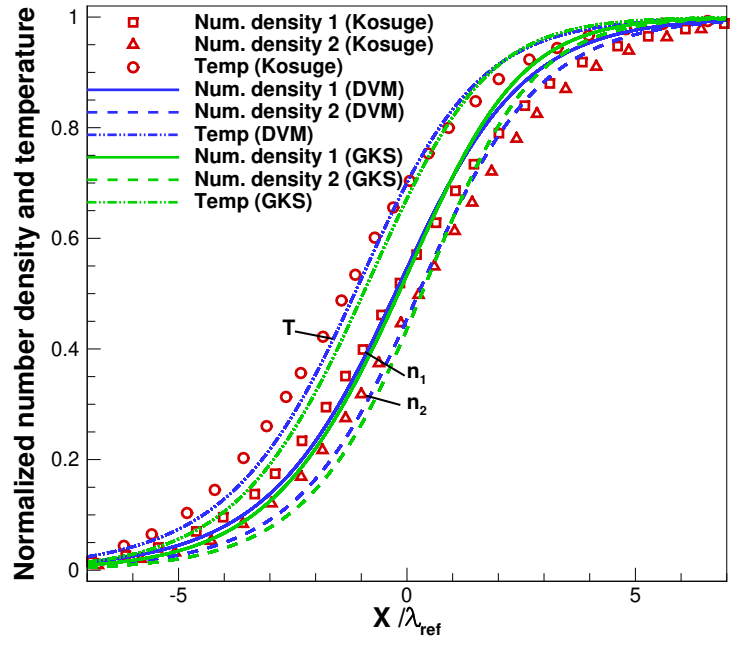

(b) $M=1.5 ; m_{2} / m_{1}=2 ; n_{1} / n=0.5$.

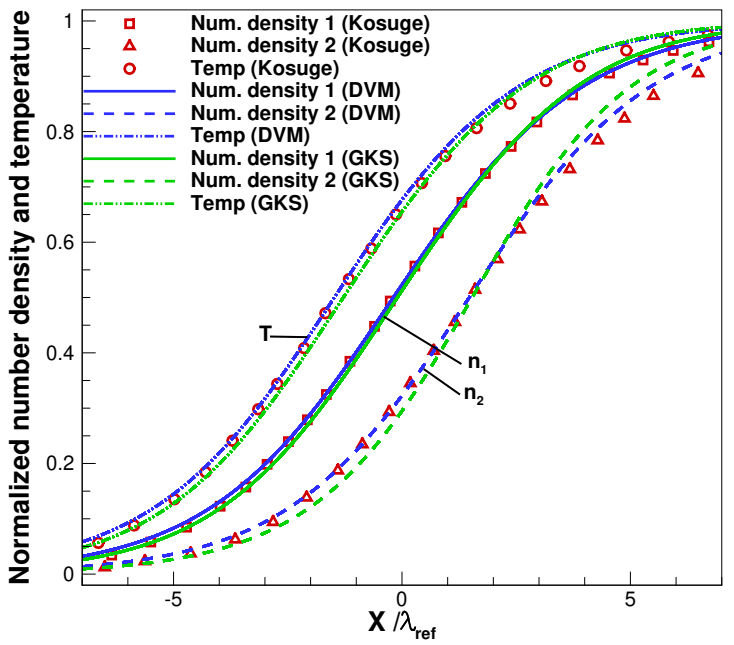

(d) $M=1.5 ; m_{2} / m_{1}=4 ; n_{1} / n=0.9 ; \omega=0.72$.

Fig. 2 Profile of species number densities and mixture temperature of a binary gas mixture through a normal shock wave for varied inflow conditions.

results (both based on the same kinetic model[25]) is to assess for what level of thermodynamic non-equilibrium the GKS approach can be employed.

Characteristic features of the problem include the lighter species (Species 1) reacting faster to the shock than the heavy species (Species 2). This becomes more evident for higher concentration of light species and with increasing mass ratios, as can be seen in Fig.2. The observed GKS solutions for the number densities and the mixture temperature match closely with the solutions from the DVM under different flow conditions. The number density of the heavy gas $\left(n_{2}\right)$, which acts as a trace gas where the concentration of light gas is $90 \%$, deviates more from the Boltzmann solution for both the DVM and GKS than the number density of the lighter gas $\left(n_{1}\right)$. The shock profile predicted by the GKS scheme 
versus the DVM is slightly steeper and as a consequence the shock is thinner in comparison to the DVM results. This thinner shock is in line with expectations based on single-species GKS results[7]. This effect will be more pronounced for higher Mach numbers. At the same time, the well-known disadvantage of the BGK-based kinetic models, i.e. the longer upstream tail, will be reduced with the steeper profile of the GKS solutions.

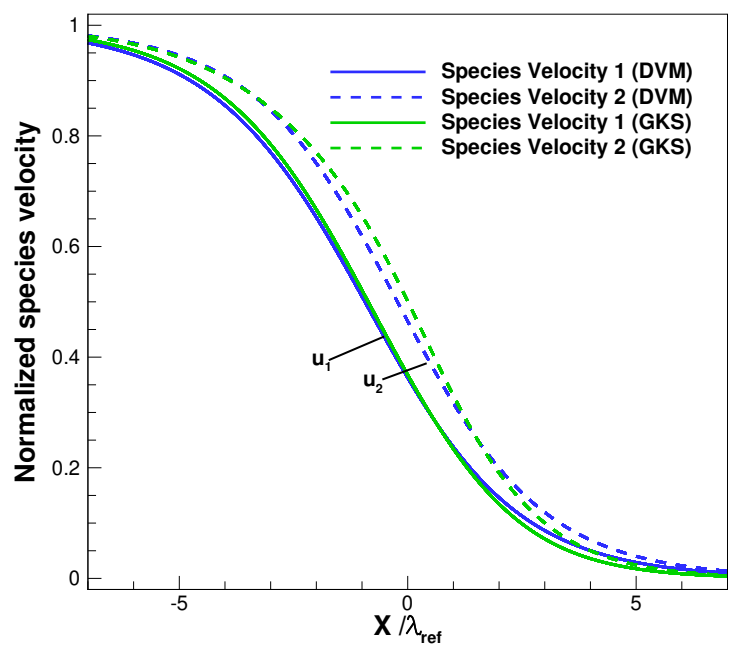

(a) $M=1.5 ; m_{2} / m_{1}=2 ; n_{1} / n=0.9$

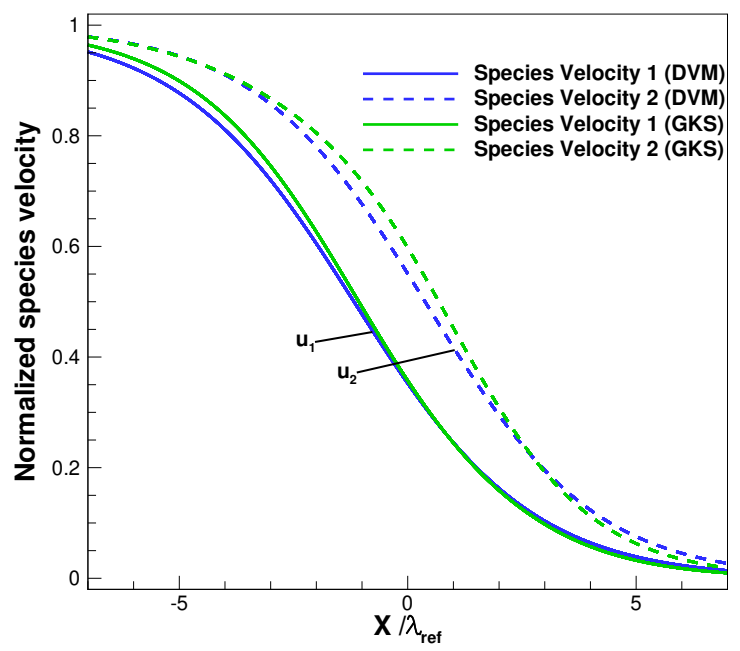

(c) $M=1.5 ; m_{2} / m_{1}=4 ; n_{1} / n=0.9$

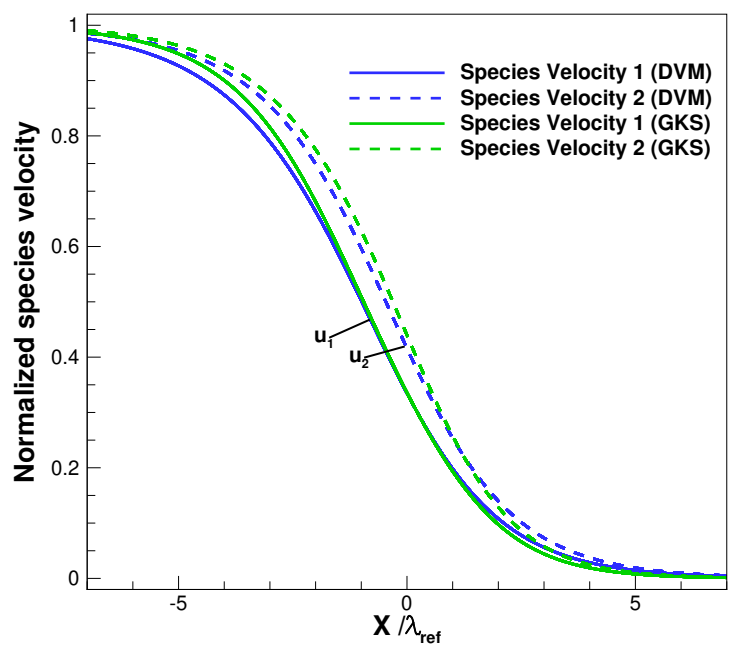

(b) $M=1.5 ; m_{2} / m_{1}=2 ; n_{1} / n=0.5$.

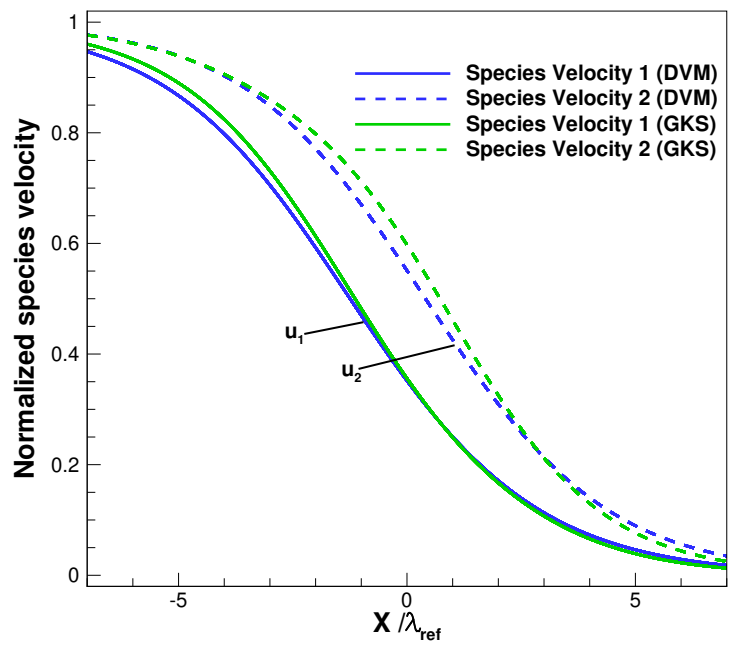

(d) $M=1.5 ; m_{2} / m_{1}=4 ; n_{1} / n=0.9 ; \omega=0.72$.

Fig. 3 Profile of species velocities of a binary gas mixture through a normal shock wave for varied inflow conditions.

The GKS presented in this paper is derived from a kinetic model that recovers three correct transport coefficients. Compared to the established GKS mixture work ([18-20]), the species mean velocities and temperatures are allowed to deviate from each other and from the gas mixture means. Figure 3 shows the normalized species velocity profiles $u_{2}$ and $u_{1}$ through the shock wave. The difference between the velocities and their change under varied conditions 
demonstrates the importance of modelling the species velocities separately. This important limitation of most existing GKS methods for gas mixtures is overcome with the current scheme. As with the number densities, the lighter species velocity reacts faster to the shock than the heavy gas. Focusing on the differences between Fig. 3 (a),(b) and (c),(d), it is easy to notice that the higher the mass ratio, the bigger the difference between the velocities, as can be expected since a stronger non-equilibrium effect will occur. The GKS provides a good match with the DVM for the solution of the species velocities. As before the shock wave profile is slightly steeper and results for the gas with bigger concentration (species 1) are in better agreement.

\section{B. Flat Plate}

A second test case considered is a supersonic rarefied flow over a flat plate. This test case is challenging due to the complex non-equilibrium boundary layer, with velocity slip as well as a jump in temperature between the wall and the gas in the immediate vicinity for the rarefied cases considered here. The changes of the flow are examined for varied species mass ratio, rarefication level and wall temperature. The DVM and GKS solutions are compared. The baseline test case is taken at $M=1.5$, mass ratio of 2 , Knudsen number 0.05 . Note that the Knudsen number is based on the reference length, calculated as in Eq. 27). The scaling factor is not required since we can compare the DVM and GKS based on the same reference length. The temperature of the wall is fixed at 1.5 times the free-stream temperature, while a fully accommodating diffuse wall is assumed. The free-stream conditions for all cases involve a concentration of $90 \%$ for the light species and 10\% heavy species. All test case conditions are summarized in the Table 2.

\section{Table 2 Test Case Conditions for a Flow Over a Flat Plate}

\begin{tabular}{cccc}
\hline$M$ & $m_{2} / m_{1}$ & $K n$ & $T_{\text {wall }}$ \\
\hline 1.5 & 2 & 0.05 & $1.5 \mathrm{~T}$ \\
1.5 & 2 & 0.025 & $1.5 \mathrm{~T}$ \\
1.5 & 4 & 0.05 & $1.5 \mathrm{~T}$ \\
1.5 & 2 & 0.05 & $1.0 \mathrm{~T}$ \\
\hline
\end{tabular}

The solutions for the flow variables for the DVM and GKS matched closely as seen from the normal shock wave results. For the same Mach number 1.5, the shock at the flat plate is weaker, which leads to even better agreement between the two numerical schemes for the macroscopic variables. On Fig. 4 the Mach number and the mixture temperature plots for the baseline test case are presented. The results demonstrate a good comparison between the DVM and GKS. We want to investigate the differences between them. Therefore, we will next focus our attention on the diffusion effects, in particular the concentration variation of the light species in the mixture (Fig. 5) and the species velocity difference $u_{2}-u_{1}$ (Fig. 6). Note that these are very detailed plots and seemingly large variations between the GKS (in color flood) and the DVM (black lines) are of the order of less than $1 \%$ for the concentrations and $3-4 \%$ for the difference in velocities. 


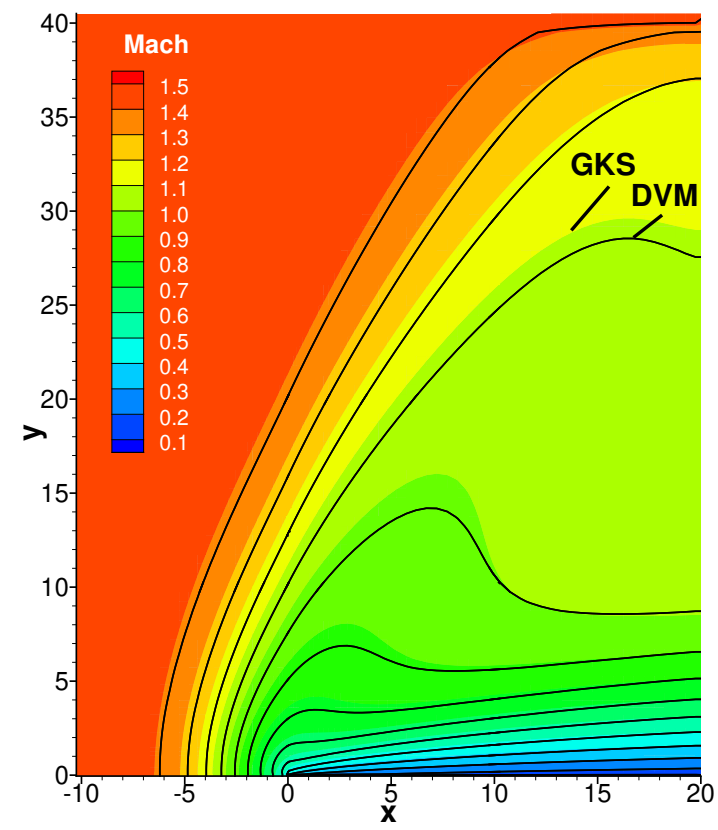

(a) Mach number

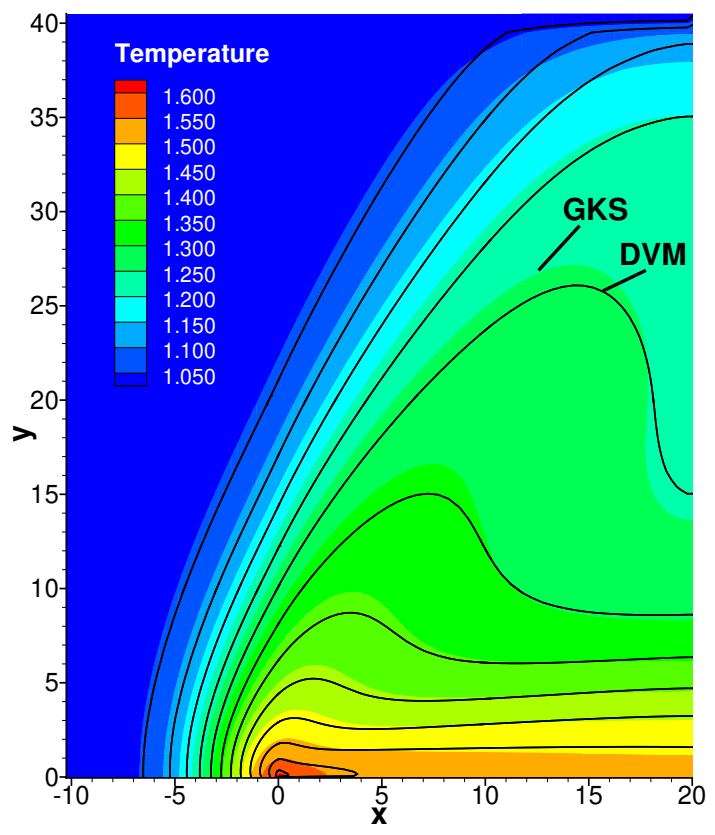

(b) Mixture temperature

Fig. 4 Mach number and mixture temperature of the baseline test case for a binary mixture flow over a flat plate for varied inflow conditions.

To highlight the effect of the Knudsen number, two different length-scales were considered in the presented numerical results. The lower-Knudsen number case involves a flat plate length of 40 reference lengths of the flow at free-stream conditions, while the higher-Knudsen number case involves a plate length of 20 reference lengths. The ratio of the species molecular masses is also varied. A further aspect investigated is the imposed wall temperature, i.e. a lower wall temperature will lead to thinner boundary layer and a somewhat weaker displacement effect.

Fig. 5 and Fig. 6 show the variation of concentration of the light species and the difference between the species velocities, respectively. The strongest non-equilibrium effects will occur close to the leading edge of the plate, where the largest gradients in the flow occur. This is evident in all the results shown in the figures. In Fig. 5 it can be seen that even for the considered flows without chemistry, the non-equilibrium effects give rise to a change in species concentration, caused by diffusion of the species. As expected the lower level of rarefaction in the $K n=0.025$ case leads to a slightly smaller change in concentration as compared to the baseline $K n=0.050$ case. Clearly, for a larger ratio of molecular masses, the velocity drift is more pronounced, leading to larger concentration changes, as can be seen in Fig. 5 (c). The effect of the lower wall temperature can be seen by comparing Fig. 55 d) with the result in Fig. 5.a). For this relatively low Mach number, it can be seen that the effect is limited, however not insignificant. Comparing the GKS and DVM results, overall a good agreement can be observed, considering the level of detail highlighted. It is important to note that the GKS solver represents concentration changes that compare well with DVM, showing that this important non-equilibrium effect is captured. 


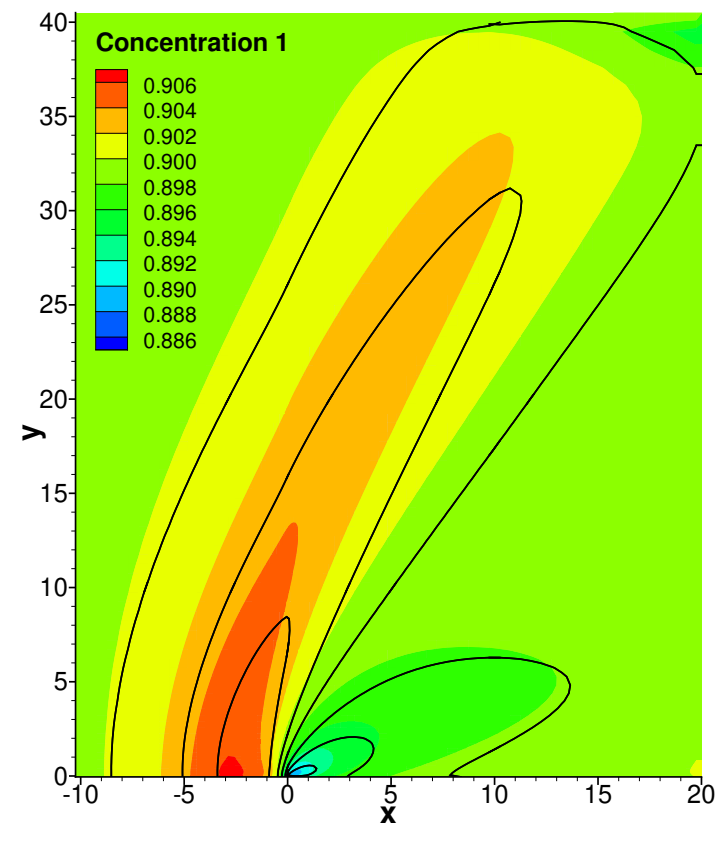

(a) $m_{2} / m_{1}=2 ; K n=0.05 ; T_{\text {wall }}=1.5 T$

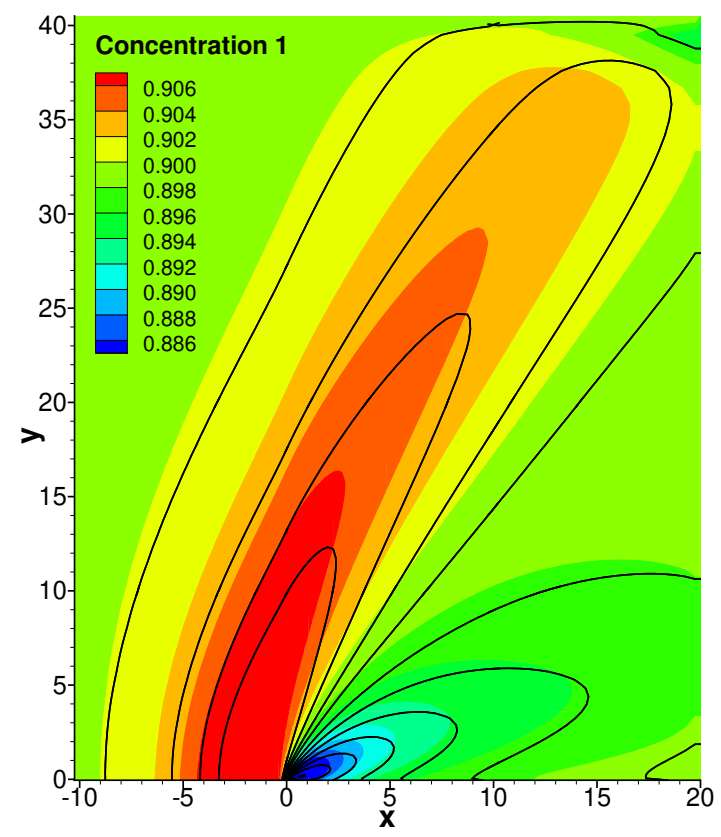

(c) $m_{2} / m_{1}=4 ; K n=0.05 ; T_{\text {wall }}=1.5 T$

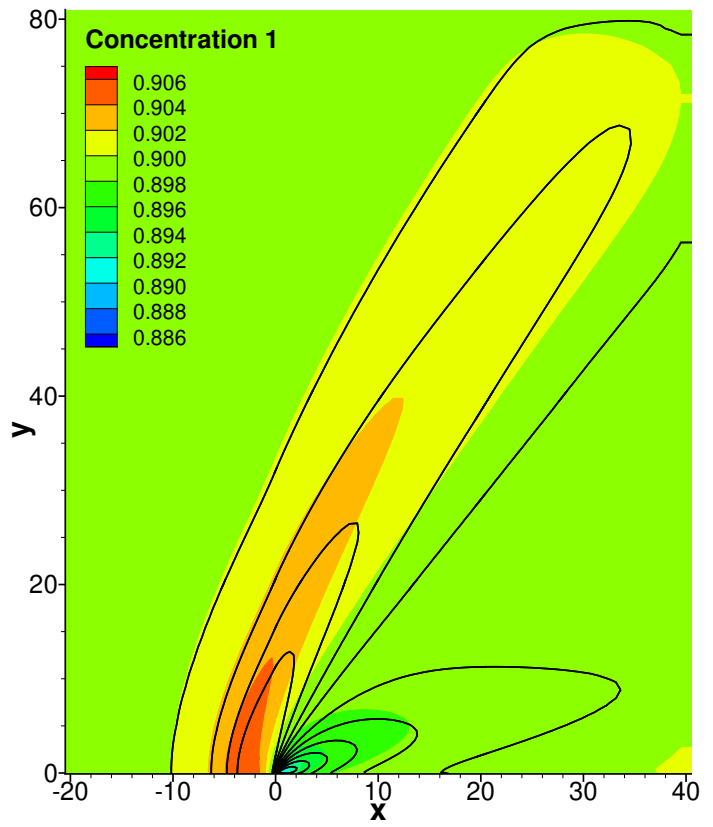

(b) $m_{2} / m_{1}=2 ; K n=0.025 ; T_{\text {wall }}=1.5 T$

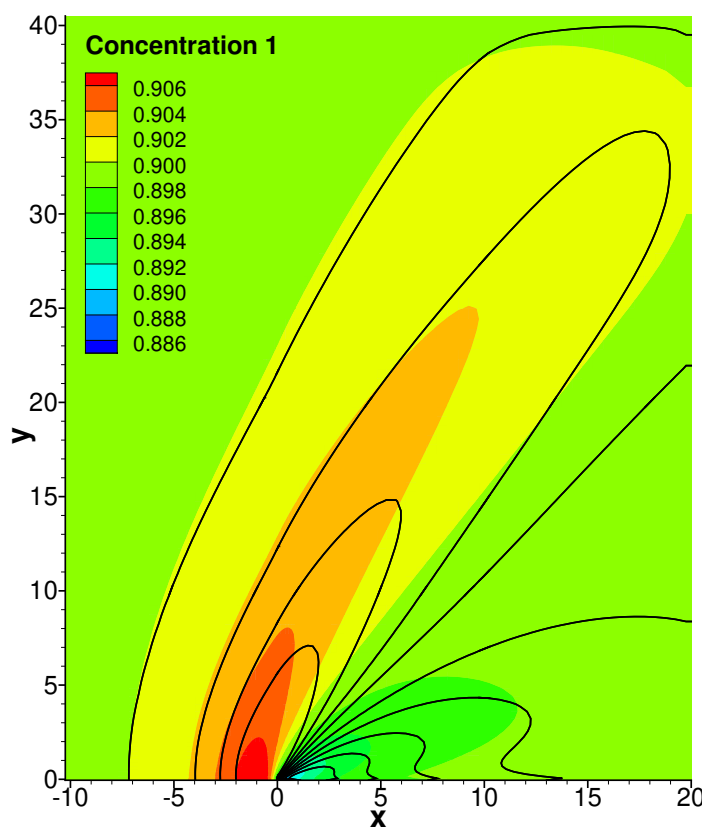

(d) $m_{2} / m_{1}=2 ; K n=0.05 ; T_{\text {wall }}=1.0 T$

Fig.5 Light species concentration variation in a binary mixture flow over a flat plate for varied inflow conditions.

A similar comparison for the different cases and for the DVM and GKS is shown in Fig. 6. The non-equilibrium effect shown here is the occurrence of velocity drift, represented by the difference between the non-dimensional species $u$-velocities. The extent of non-equilibrium effects follows the pattern of that in the concentration changes, i.e. with more pronounced effects for larger $K n$ and mass ratio, mainly focussed in the immediate vicinity of the plate leading 

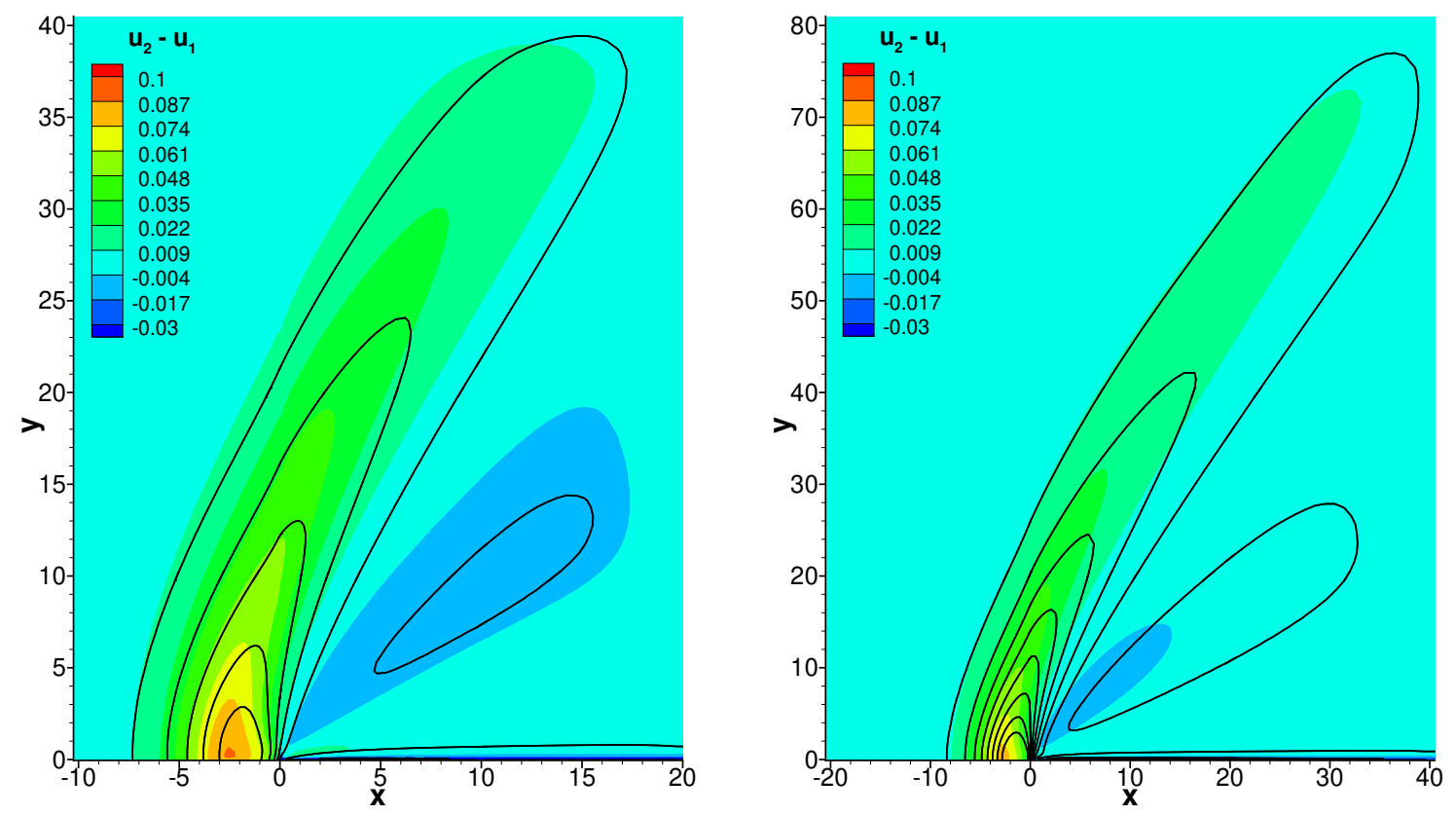

(a) $m_{2} / m_{1}=2 ; K n=0.05 ; T_{\text {wall }}=1.5 T$

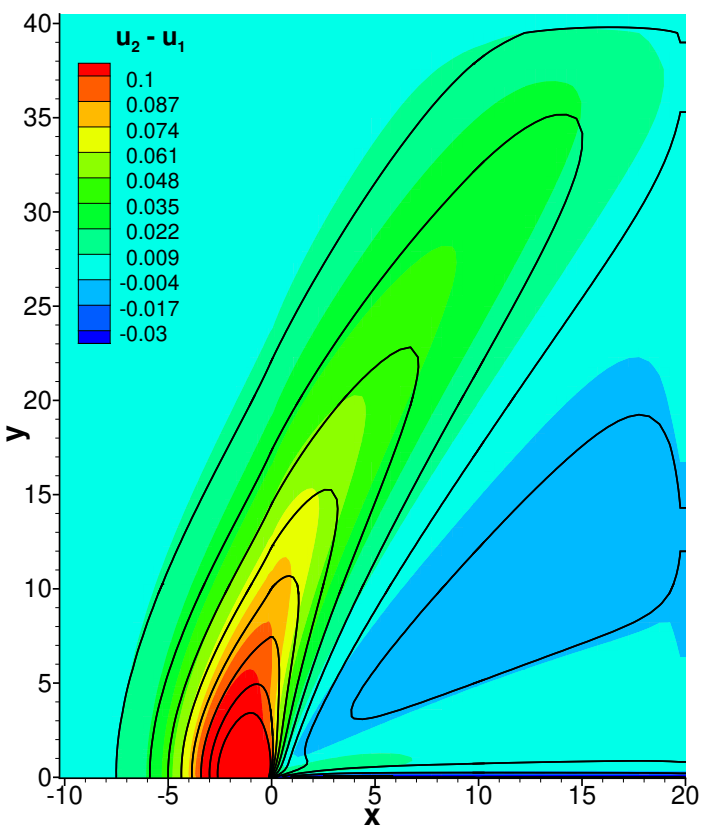

(b) $m_{2} / m_{1}=2 ; K n=0.025 ; T_{\text {wall }}=1.5 T$

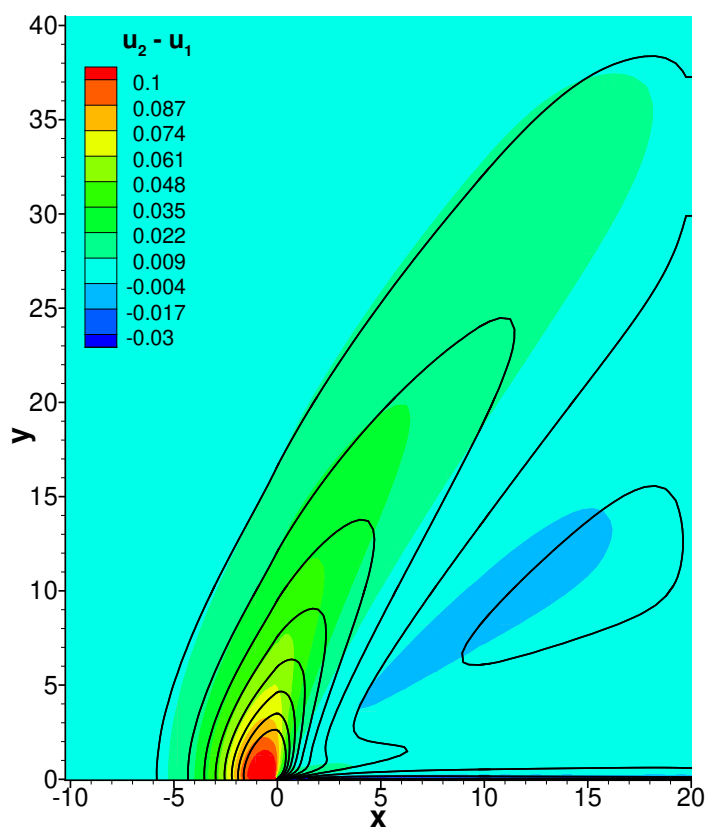

(c) $m_{2} / m_{1}=4 ; K n=0.05 ; T_{\text {wall }}=1.5 T$

(d) $m_{2} / m_{1}=2 ; K n=0.05 ; T_{\text {wall }}=1.0 T$

Fig. 6 Non-dimensional species mean velocity difference $u_{2}-u_{1}$ in a binary mixture flow over a flat plate for varied inflow conditions.

edge. Comparing Fig.6(a) and Fig. 6(c), it shows that the maximum and the region of the difference between the species velocities are bigger for the larger mass ratio case.

In order to explore the limitations of the gas-kinetic scheme, we focus on the shear stress at the wall of the flat 
plate. The biggest variation between the DVM and the GKS is at the leading edge of the flat plate and in the cells just above it. Three test cases are considered with fixed Mach number 1.5, mass ratio 2 and light species concentration $90 \%$, while the Knudsen number is varied. Starting at the baseline test case at $K n=0.05$ (in blue), we consider a more rarefied flow at $K n=0.075$ (in green) and a more continuum flow at $K n=0.0125$ (in red). On Fig. 7(a) the shear stress along the flat plate is presented. As expected the biggest variation between the DVM (square symbols) and GKS(delta symbols) solutions is at the leading edge of the flat plate. The most rarefied case with $K n=0.075$ shows the biggest difference between the DVM and GKS in the result for the shear stress. As the flow becomes more continuum towards Knudsen number of 0.05 and 0.0125 this difference decreases. Since the GKS is based on the Chapman-Enskog and as demonstrated in this plot, the results will keep improving as $K n \rightarrow 0$ (in the limit of the hydrodynamic limit).

Focusing on the peak value of the shear stress along the flat plate, the velocity profile for the different Knudsen numbers is plotted at that point on Fig. 7 (b). The velocity profile is well-captured, but the difference between the two schemes at the plate propagates up through the whole profile. It should be noted that the more rarefied cases seem to match the two gas mixture velocities better, but this is only due to the scaling of the problem. The same location on the y-axis on Fig. 7(b), e.g. $y=20 L_{r e f}$, when compared to each flat plate length of $80 L_{r e f}(K n=0.0125), 20 L_{r e f}(K n=0.05), 13.3 L_{r e f}(K n=0.075)$ refers to a different location at the farfield of each case. Therefore, the velocity of the most rarefied case $(K n=0.075)$ in fact takes the longest to converge to the same value.

The profile of the shear stress is plotted at two different points. One is at the peak of the stress along the plate on

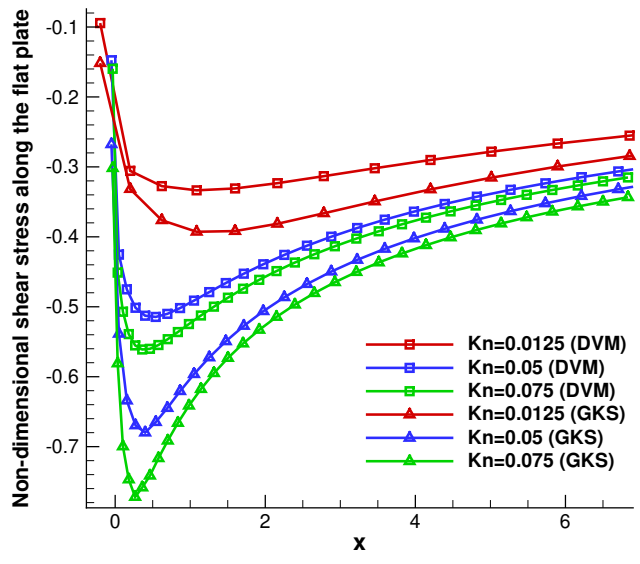

(a) Shear Stress along the Flat Plate

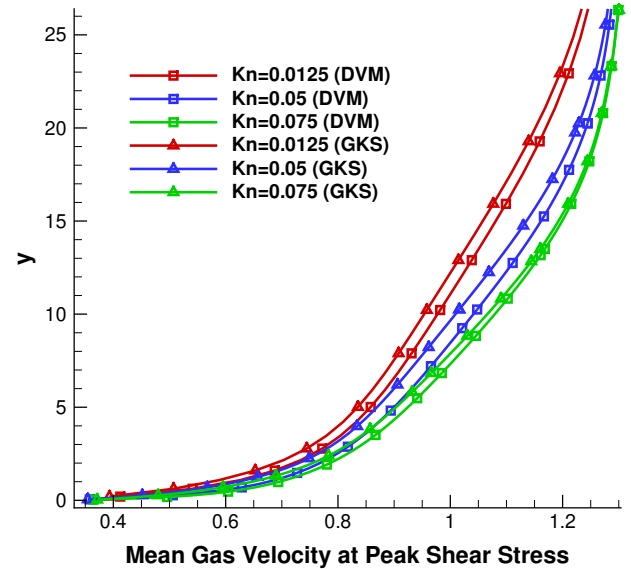

(b) Velocity Profile at Peak Value of Shear Stress

Fig. 7 Shear Stress and Velocity Profile at the Flat Plate

Fig. 7(b) and the other is further downstream: at a quarter of each plate's length - on Fig. 8.b) to establish how the flow develops. At the peak shear stress location the variation between the DVM (square) and GKS (delta) values of 
the shear stress in the first few cells is significant. The biggest difference is for the most rarefied case, where the GKS overpredicts the value of the shear stress in the vicinity of the wall. The difference propagates in the farfield but reduces quickly. The match between profiles of the shear stress at the downstream location from the GKS and DVM is much better as shown on ig. 8 b). The most rarefied test case shows good agreement between the schemes, while the test case at $(K n=0.0125)$ demonstrates the convergence of the GKS when the flow goes towards the continuum limit.

From these results and the shown comparison between DVM and GKS it follows that despite the relatively large

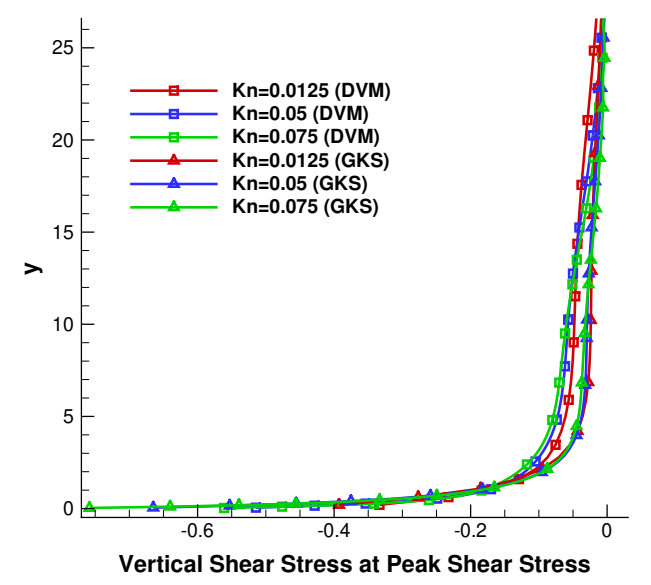

(a) Shear Stress Profile at the Peak Shear

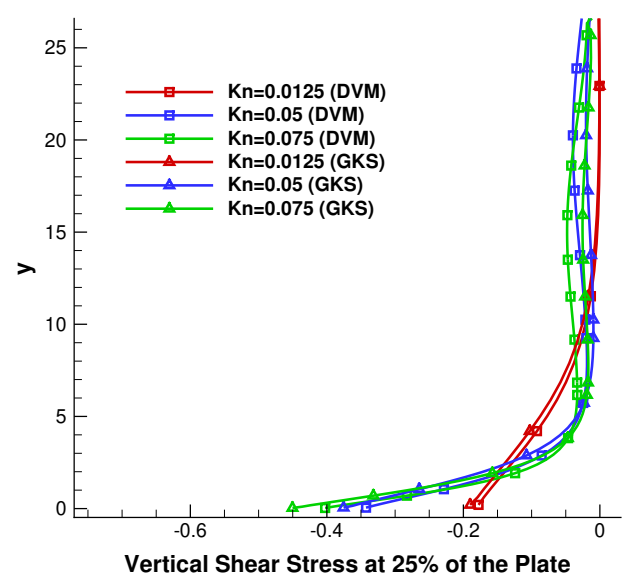

(b) Shear Stress at a Quarter of the Plates' Lengths

Fig. 8 Vertical Shear Stress Profile at Two Locations

Knudsen numbers considered for this flat plate case, the GKS performs relatively well. Partly this can be explained by the Mach number of 1.5. For higher Mach numbers, stronger non-equilibrium will occur and bigger deviations can be expected. It should be noted that currently the GKS is limited at Mach number of 1.5 and Knudsen numbers up to 0.05 . For this reason they have been extensively investigated in this paper. It was also found that Mach number of 2 and $\mathrm{Kn}=0.1$ are critical for the GKS at concentration of $90 \%$. A more detailed investigation of this aspect forms part of future work.

In the next subsection the main advantage of the GKS - the computational efficiency - is discussed.

\section{Computational Efficiency}

The described results were achieved by implementing the new mixture kinetic model with a DVM and a GKS scheme. The distribution functions are per species and vary with the mass of the each gas. For higher mass ratios $m_{2} / m_{1}$ for the DVM the number of discrete velocities needs to be increased in order to correctly resolve the velocity distribution functions. This is also true for an increase in the Mach number. While for a one-dimensional test the increase of velocities is possible, it becomes computationally highly expensive to achieve the same resolution in higher 
dimensions. The main goal for constructing the gas-kinetic scheme is achieving numerical efficiency, which will allow for more complex cases and aerospace vehicles to be investigated. The results for a normal shock and the supersonic flow over a flat plate under different conditions demonstrated that the GKS is capable of preserving the physical details of the flow. There is a good agreement between the solutions from the discrete velocity method and the gas-kinetic scheme based on the same kinetic model for the cases considered. The shock wave grid has 1200 cells in the $\mathrm{x}$-direction for the DVM and GKS simulations. Two-dimensional formulations were used for the flat plate simulations. The flat plate's grid is three-dimensional and has 19200 cells, but no computational effort is used in the third dimension. By comparing the computational requirements for the shock wave and flat plate problems, we observe the effect of higher dimensionality for both the DVM and GKS simulations. For the shock wave DVM test case 100 uniformly spaced discrete velocity are sufficient, while for the flat plate DVM $32 \times 32=1024$ discrete velocities were used. Note the number of discrete velocities for the flat plate are limited and provide a relatively course velocity grid. Notice if we have used the same number of discrete velocities as for the shock wave but in two dimensions, the flat plate test case would run with $100 \times 100=10000$, which would increase the CPU time for the DVM significantly. The simulations were run on a quad-core Intel ${ }^{\circledR X}$ Xeon ${ }^{\circledR} 3.30 \mathrm{GHz}$ computer. Table 3 demonstrates the CPU time required for every 1000 steps for the DVM and the GKS for the two baseline test cases: $M=1.5, m_{2} / m_{1}=2, n_{1} / n=0.9$ and for the flat plate also $K n=0.05, T_{\text {wall }}=1.5 T$. The times quoted are those for simulations running on a single core.

Table 3 CPU Time per 1000 Steps

\begin{tabular}{ccc}
\hline & Shock Wave (1D) & Flat Plate (2D) \\
\hline DVM & $64 \mathrm{~min}$ & $376 \mathrm{~min}$ \\
GKS & $22.7 \mathrm{sec}$ & $1 \mathrm{~min} 15 \mathrm{sec}$ \\
Speed-up & 169 & 301 \\
\hline
\end{tabular}

The orders of magnitude difference between the CPU time of the DVM and the GKS methods is very significant. The differences between the relative speed-ups for the shock wave and the flat-plate cases are due to the fact that in the flat-plate DVM a two-dimensional velocity-space discretization was used, while for the DVM simulations of the shock wave, a one-dimensional discretization was employed. The CPU time difference for the GKS shock wave and flat plate simulations is directly the result of the larger mesh for the flat-plate case, while the difference between the DVM simulations results from both the larger mesh and the increased number of discrete velocities. For a larger Mach number or species mass ratio, the DVM approach requires an increased number of discrete velocities, leading to significantly larger CPU times, while the CPU time for the GKS remains the same. Overall, the GKS method shows a significant computational advantage and promising numerical results. 


\section{Conclusion}

A new gas-kinetic scheme was introduced for a binary mixture of monoatomic gases based on a new kinetic model, which provides the correct transport properties in the continuum limit, i.e. recovering the correct viscosity, heat flux and diffusion coefficients. This model is more advanced and detailed than the kinetic models previously used as the basis for GKS methods. Using a DVM implementation, the model was assessed for a range of high-speed test cases, showing that species diffusion effects are captured in detail in regions with strong non-equilibrium. Based on this model, the derivation of a GKS was detailed, followed by the application to the high-speed test cases, previously considered for the DVM. The GKS method agrees well with the DVM method for the cases of a shock wave and a flow past a flat plate. Further work will include more demanding test cases, e.g. higher Mach numbers and different geometries. The good comparison with DVM results, together with the computational efficiency, make this GKS scheme a viable alternative for supersonic mixture flows in the moderately rarefied regime. The range of applications for which the presented GKS scheme can be effectively used will be investigated further in future work. Furthermore, the present work will be extended with kinetic models for diatomic gases. This will greatly increase the range of aerospace applications that can be efficiently modeled with the proposed GKS approach.

\section{Acknowledgments}

The authors would like to acknowledge the financial support provided by the University of Glasgow. Results were obtained using the EPSRC funded ARCHIE-WeSt High Performance Computer (www.archie-west.ac.uk). EPSRC grant no. EP/K000586/1.

\section{References}

[1] Chapman, S., and Cowling, T., The Mathematical Theory of Non-Uniform Gases, $2^{\text {nd }}$ ed., Cambridge University Press,London, 1952.

[2] Bhatnagar, P. L., Gross, E. P., and Krook, M., "A Model for Collision Processes in Gases. I. Small Amplitude Processes in Charged and Neutral One-Component Systems," Physical Review, Vol. 94, 1954, pp. 511-525. doi:10.1103/PhysRev.94.511.

[3] Shakhov, E. M., “Generalization of the Krook kinetic relaxation equation,” Fluid Dynamics, Vol. 3, No. 5, 1968 , pp. 95-96. doi:10.1007/BF01029546.

[4] Holway, L. H., "New Statistical Models for Kinetic Theory: Methods of Construction,” The Physics of Fluids, Vol. 9, No. 9, 1966, pp. 1658-1673. doi:10.1063/1.1761920.

[5] Bird, G., Molecular Gas Dynamics and the Direct Simulation of Gas Flows, Oxford Science Publications, 1994.

[6] Sanders, R., and Prendergast, K., "The possible relation of the 3-kiloparsec arm to explosions in the galactic nucleus," The Astrophysical Journal, Vol. 188, 1974, pp. 489-500. doi:10.1086/152739. 
[7] Colonia, S., Steijl, R., and Barakos, G., "Kinetic models and gas-kinetic schemes for hybrid simulation of partially rarefied flows," AIAA Journal, Vol. 54, No. 4, 2016, pp. 1264-1276. doi:10.2514/1.J054137.

[8] Prendergast, K. H., and Xu, K., "Numerical Hydrodynamics from Gas-Kinetic Theory,” Journal of Computational Physics, Vol. 109, No. 1, 1993, pp. 53 - 66. doi:10.1006/jcph.1993.1198.

[9] Xu, K., and Prendergast, K. H., "Numerical Navier-Stokes Solutions from Gas Kinetic Theory,” Journal of Computational Physics, Vol. 114, No. 1, 1994, pp. 9 - 17.

[10] Xu, K., "A Gas-Kinetic BGK Scheme for the Navier-Stokes Equations and Its Connection with Artificial Dissipation and Godunov Method,” Journal of Computational Physics, Vol. 171, No. 1, 2001, pp. 289 - 335. doi:10.1006/jcph.2001.6790.

[11] Xu, K., Mao, M., and Tang, L., “A multidimensional gas-kinetic BGK scheme for hypersonic viscous flow,” Journal of Computational Physics, Vol. 203, No. 2, 2005, pp. 405 - 421. doi:10.1016/j.jcp.2004.09.001.

[12] Xu, K., and Huang, J., "A unified gas-kinetic scheme for continuum and rarefied flows," Journal of Computational Physics, Vol. 229, No. 20, 2010, pp. 7747 - 7764. doi:10.1016/j.jcp.2010.06.032.

[13] Huang, J.-C., Xu, K., and Yu, P., “A Unified Gas-Kinetic Scheme for Continuum and Rarefied Flows II: Multi-Dimensional Cases," Communications in Computational Physics, Vol. 12, No. 3, 2012, p. 662-690. doi:10.4208/cicp.030511.220911a.

[14] Liu, S., Yu, P., Xu, K., and Zhong, C., "Unified gas-kinetic scheme for diatomic molecular simulations in all flow regimes," Journal of Computational Physics, Vol. 259, 2014, pp. 96 - 113. doi:10.1016/j.jcp.2013.11.030.

[15] Wang, Z., Yan, H., Li, Q., and Xu, K., "Unified gas-kinetic scheme for diatomic molecular flow with translational, rotational, and vibrational modes," Journal of Computational Physics, Vol. 350, 2017, pp. 237 - 259. doi:10.1016/j.jcp.2017.08.045.

[16] Guo, Z., Xu, K., and Wang, R., "Discrete unified gas kinetic scheme for all Knudsen number flows: Low-speed isothermal case," Phys. Rev. E, Vol. 88, 2013, p. 033305. doi:10.1103/PhysRevE.88.033305.

[17] Guo, Z., Wang, R., and Xu, K., "Discrete unified gas kinetic scheme for all Knudsen number flows. II. Thermal compressible case," Phys. Rev. E, Vol. 91, 2015, p. 033313. doi:10.1103/PhysRevE.91.033313.

[18] Xu, K., "BGK-Based Scheme for Multicomponent Flow Calculations," Journal of Computational Physics, Vol. 134, No. 1, 1997, pp. 122 - 133. doi:10.1006/jcph.1997.5677.

[19] Lian, Y., and Xu, K., "A Gas-Kinetic Scheme for Multimaterial Flows and Its Application in Chemical Reactions,” Journal of Computational Physics, Vol. 163, No. 2, 2000, pp. 349-375. doi:10.1006/jcph.2000.6571.

[20] Pan, L., Cheng, J., Wang, S., and Xu, K., “A Two-Stage Fourth-Order Gas-Kinetic Scheme for Compressible Multicomponent Flows," Communications in Computational Physics, Vol. 22, No. 4, 2017, p. 1123-1149. doi:10.4208/cicp.OA-2017-0023.

[21] Wang, R., and Xu, K., "Unified gas-kinetic scheme for multi-species non-equilibrium flow," Advances in Applied Mathematics and Mechanics, Vol. 7, No. 2, 2014, p. 245-266. doi:10.1063/1.4902698. 
[22] Andries, P., Aoki, K., and Perthame, B., “A Consistent BGK-Type Model for Gas Mixtures,” Journal of Statistical Physics, Vol. 106, No. 5, 2002, pp. 993-1018. doi:10.1023/A:1014033703134.

[23] Zhang, Y., Zhu, L., Wang, R., and Guo, Z., "Discrete unified gas kinetic scheme for all Knudsen number flows. III. Binary gas mixtures of Maxwell molecules,” Phys. Rev. E, Vol. 97, 2018, p. 053306. doi:10.1103/PhysRevE.97.053306.

[24] Zhang, Y., Zhu, L., Wang, P., and Guo, Z., "Discrete unified gas kinetic scheme for flows of binary gas mixture based on the McCormack model," Physics of Fluids, Vol. 31, No. 1, 2019, p. 017101. doi:10.1063/1.5063846.

[25] Todorova, B., and Steijl, R., "Derivation and numerical comparison of Shakhov and Ellipsoidal Statistical kinetic models for a monoatomic gas mixture," European Journal of Mechanics - B/Fluids, Vol. 76, 2019, pp. 390 - 402. doi:10.1016/j.euromechflu. 2019.04.001.

[26] Todorova, B., and Steijl, R., "Discrete-velocity simulations of high-speed flows based on binary gas mixture kinetic models," (not yet published, Rarefied Gas Dynamics (RGD) conference, Glasgow, UK), 2018.

[27] Kosuge, S., Aoki, K., and Takata, S., "Shock-wave structure for a binary gas mixture: finite-difference analysis of the Boltzmann equation for hard-sphere molecules," European Journal of Mechanics - B/Fluids, Vol. 20, No. 1, 2001 , pp. 87 - 126. doi:10.1016/S0997-7546(00)00133-3.

[28] White, C., Borg, M., Scanlon, T., Longshaw, S., John, B., Emerson, D., and Reese, J., “dsmcFoam+: An OpenFOAM based direct simulation Monte Carlo solver," Computer Physics Communications, Vol. 224, 2018, pp. 22 - 43. doi:10.1016/j.cpc.2017.09.030.

[29] Vincenti, W., and Kruger, C., Introduction to physical gas dynamics, $2^{\text {nd }}$ ed., John Wiley and Sons, New York, 1967.

[30] Groppi, M., Monica, S., and Spiga, G., “A kinetic ellipsoidal BGK model for a binary gas mixture,” EPL (Europhysics Letters), Vol. 96, No. 6, 2011, p. 64002. doi:10.1209/0295-5075/96/64002.

[31] Weissman, S., and Mason, E. A., "Determination of gaseous-diffusion Coefficients from Viscosity Measurements," The Journal of Chemical Physics, Vol. 37, No. 6, 1962, pp. 1289-1300. doi:10.1063/1.1733277.

[32] Garzo, V., Santos, A., and Brey, J. J., “A kinetic model for a multicomponent gas,” Physics of Fluids A: Fluid Dynamics, Vol. 1, No. 2, 1989, pp. 380-383. doi:10.1063/1.857458.

[33] Brull, S., "An Ellipsoidal Statistical Model for gas mixtures," Communications in Mathematical Sciences, Vol. 13, No. 1, 2015, pp. 1 - 13. doi:10.4310/CMS.2015.v13.n1.a1.

[34] Center, R. E., "Measurement of Shock Wave Structure in Helium Argon Mixtures," The Physics of Fluids, Vol. 10, No. 8, 1967, pp. 1777-1784. doi:10.1063/1.1762357.

[35] Harnett, L. N., and Muntz, E. P., "Experimental Investigation of Normal Shock Wave Velocity Distribution Functions in Mixtures of Argon and Helium,” The Physics of Fluids, Vol. 15, No. 4, 1972, pp. 565-572. doi:10.1063/1.1693949. 